\title{
Incorporation of Stochastic Policyholder Behavior in Analytical Pricing of GMABs and GMDBs
}

\author{
Marcos Escobar ${ }^{1}$, Mikhail Krayzler ${ }^{2}$, Franz Ramsauer ${ }^{3, *}$, David Saunders ${ }^{4}$ and Rudi Zagst ${ }^{3}$ \\ 1 Department of Statistical and Actuarial Sciences, Western University, 1151 Richmond Street, London, \\ ON N6A 5B7, Canada; marcos.escobar@uwo.ca \\ 2 risklab GmbH, Allianz Global Investors, Seidlstraße 24-24a, 80335 Munich, Germany; \\ mikhail.krayzler@allianzgi.com \\ 3 Chair of Mathematical Finance, Technical University of Munich, Parkring 11, 85748 Garching-Hochbrück, \\ Germany; zagst@tum.de \\ 4 Department of Statistics and Actuarial Science, University of Waterloo, 200 University Avenue West, \\ Waterloo, ON N2L 3G1, Canada; dsaunders@uwaterloo.ca \\ * Correspondence: franz.ramsauer@tum.de; Tel.: +49-89-289-17417; Fax: +49-89-289-17407
}

Academic Editor: Pavel Shevchenko

Received: 5 September 2016; Accepted: 1 November 2016; Published: 8 November 2016

\begin{abstract}
Variable annuities represent certain unit-linked life insurance products offering different types of protection commonly referred to as guaranteed minimum benefits (GMXBs). They are designed for the increasing demand of the customers for private pension provision. In this paper we analytically price variable annuities with guaranteed minimum repayments at maturity and in case of the insured's death. If the contract is prematurely surrendered, the policyholder is entitled to the current value of the fund account reduced by the prevailing surrender fee. The financial market and the mortality model are affine linear. For the surrender model, a Cox process is deployed whose intensity is given by a deterministic function (s-curve) with stochastic inputs from the financial market. So, the policyholders' surrender behavior depends on the performance of the financial market and is stochastic. The presented pricing scheme incorporates the stochastic surrender behavior of the policyholders and is only based on suitable closed-form approximations.
\end{abstract}

Keywords: variable annuities; surrender behavior; closed-form approximation; pricing; affine linear model

\section{Introduction}

Variable annuity (VA) contracts represent a "wide range of life insurance products, whose benefits can be protected against investment and mortality risks by selecting one or more guarantees" [1]. Since VAs are usually unit-linked, they allow policyholders to participate in rising stock prices while their guarantees offer protection against the reverse trend. For further reading on VAs and implicit options embedded in general life insurance products, see [2-4]. In contrast to the policyholders, for VA providers the GMXBs that are offered may cause severe financial and actuarial risks: First, the minimum benefits could expire in-the-money, i.e., worth more than the corresponding position in stocks. Second, there might be a difference between the expected and the realized mortality rates.

Furthermore, VA providers are also exposed to behavioral risk, which in this context is often referred to as surrender or lapse risk. This is the risk that the policyholders cancel their contracts in a manner different from the assumptions made by the VA provider. Longevity risk can be modeled independently from financial risk, whereas surrender risk substantially depends on the evolution of the financial markets. For example, increasing interest rates might lead to increasing cancellation rates, as alternative investment products with a higher guaranteed rate or at a cheaper price will appear. 
Modeling policyholder risk should deserve special attention, as "it has influence on the pricing of the options and guarantees within the contracts, on solvency capital requirements, and hedging effectiveness" [5]. High losses have been reported by VA carriers due to changes in surrender assumptions (Mountain Life reported an increase in the value of its liabilities by USD 48 bn due to a reduction of assumed surrender rates ${ }^{1}$ ). Policyholder risk is not hedgeable, might lead to severe liquidity problems, and, therefore, needs to be analyzed and priced carefully. In this paper we focus on this type of risk; we do not claim to find the precise relationship between surrender rates and economic factors (internal or external), but rather show how the well-known patterns can be included in the pricing framework suggested by [6]. Furthermore, as opposed to the work of [6], the market price of mortality risk is explicitly taken into account.

In the last decade, several empirical studies appeared analyzing the main drivers of policy cancellations. From the perspective of a classical life insurance business, two major hypotheses can be differentiated: the interest-rate hypothesis and the emergency fund hypothesis. The first one, advanced by, e.g., $[7,8]$ (especially in the long run), assumes that an increase in interest rates leads to an increase in surrender rates. This is explainable by the fact that higher interest rates generally lead to higher annuity rates within other similar decumulation products and, hence, policyholders have an incentive to cancel their existing VA and enter a new one. In the VA business, high interest rates lead to either higher guaranteed benefits for the same guarantee price or to the same guaranteed benefits but for lower prices. The second hypothesis, empirically supported, e.g., by [9], assumes that policyholders might need to terminate their life or pension insurances due to a personal financial distress. To model this dependency, most of the papers use macroeconomic risk factors assuming that the general state of the economy serves as a proxy for personal financial circumstances. Some of the papers support both hypotheses, see, e.g., [10] for an analysis of the Korean case or [11] for the U.S. life insurance market.

As variable annuities depend on the performance of financial markets, apart from the interest-rate and the emergency fund hypotheses driving policyholder behavior within traditional life insurance business, the so-called moneyness hypothesis has been the focus of several recent studies. This concept relies on the fact that the value of the guarantee (approximated by the moneyness defined as the ratio of the surrender to the guaranteed value) should have a substantial impact on policyholders' decisions. That is, the better the performance of a fund underlying the VA product, the higher the moneyness of the contract and, consequently, the lower the economic value of the guarantee. Therefore, there is a significant incentive for the insured person to cancel the contract and potentially enter a new one with a higher guaranteed value. Empirical evidence for this hypothesis is given, for example, in $[5,12]$. The former paper also tests and supports interest-rate and emergency fund hypotheses in the context of variable annuities. According to the company surveys conducted by $[5,13]$ as well as shown in some examples of [7], policyholders do not always act rationally. They cancel their products even when it is not economically rational ${ }^{2}$ and also do not surrender their guarantees deep out of the money.

Additionally, there is abundant literature on the incorporation of lapse behavior in pricing models. We provide here just a short overview of the main papers in that area and refer interested readers to [14] for a broad classification of lapse rate models. According to their work, one can differentiate between three major groups of papers depending on the assumptions made on the policyholder rationality.

First, pure dynamic surrender models assume optimal cancellation for risk-neutral investors [15-19]. These authors interpret the surrender option as an American option and provide numerical solutions for optimal stopping problems to determine its price. The second group of papers assumes optimal dynamic lapsation for rational and risk-averse investors [20,21]. The authors assume that policyholders maximize their expected utility, which is modeled via a constant relative risk

1 Source: White Mountain Insurance Group Report 2010. In this case the expected number of policyholders entitled to the final payoff increases and therefore, the present value of liabilities rises as well.

2 This is more or less a general assessment. Cancellation could be rational and utility-maximizing for specific policyholders, however, these personal reasons for cancellation are not included in the model. 
aversion (CRRA) function. The third group of papers eschews the assumption of optimal policyholder behavior and rather tries to incorporate the above mentioned empirical evidence in dynamic lapse modeling. Examples of these papers include [2,22-26].

Our work also belongs to the third group of papers. The contributions to the literature are as follows. First, instead of the stand-alone consideration of different stylized facts, we explicitly incorporate the moneyness and interest-rate hypotheses in our hybrid pricing framework at the same time; Second, we include the emergency fund hypothesis in the model; Finally, we derive analytical approximations for the selected guarantees under financial, actuarial, and behavioral risks. In this paper we concentrate on Guaranteed Minimum Accumulation, Death, and Surrender Benefits. Extension of the suggested approach for the pricing of other variable annuity products constitutes one of the directions of future research.

The remainder of the paper is structured as follows: The second section describes the stochastic models for the financial market, the insureds' mortality, and the policyholders' surrender behavior. The third section specifies the considered type of VAs and derives the closed-form approximations. The fourth section shows how the models of the second section can be calibrated using actively traded products and historical mortality tables. The fifth section presents an example of the pricing scheme. The sixth section discusses extensions to the surrender model. The seventh section provides conclusions and possible directions for future research.

\section{Stochastic Models}

\subsection{Financial Market Model}

Let $(\Omega, \mathcal{F}, \mathbb{F}, \mathbb{Q})$ be a filtered probability space with risk-neutral pricing measure $\mathbb{Q}$ and filtration $\mathbb{F}:=\left(\mathcal{F}_{t}\right)_{t \geq 0}$ satisfying the usual conditions, i.e., the filtration is right-continuous and $\mathcal{F}_{0}$ is saturated. Furthermore, let the instantaneous interest-rate process $r:=(r(t))_{t \geq 0}$ and the stock price process $S:=(S(t))_{t \geq 0}$ be given by the Hull-White extended Vasicek model [27] and a generalized geometric Brownian motion with stochastic drift $r$. For any point in time $t \geq 0$ the stochastic process $Y:=(Y(t))_{t \geq 0}$ defined by $Y(t):=\ln (S(t) / S(0))$ represents the accumulated log-return up to $t$. If the constant $\rho_{S r} \in[-1 ; 1]$ denotes the correlation between the Brownian motions of the processes $r$ and $S$, we end up with the following risk-neutral financial market:

$$
\begin{aligned}
\mathrm{d} r(t) & =\left(\theta_{r}(t)-a_{r} r(t)\right) \mathrm{d} t+\sigma_{r} \mathrm{~d} W_{r}^{\mathbb{Q}}(t), \\
\mathrm{d} Y(t) & =\left(r(t)-\frac{1}{2} \sigma_{S}^{2}(t)\right) \mathrm{d} t+\sigma_{S}(t) \mathrm{d} W_{S}^{\mathbb{Q}}(t), \\
r(0) & =r_{0}, Y(0)=0, \mathrm{~d} W_{S}^{\mathbb{Q}}(t) \mathrm{d} W_{r}^{\mathbb{Q}}(t)=\rho_{S r} \mathrm{~d} t .
\end{aligned}
$$

To derive the closed-form approximation in Section $3.2 a_{r}$ and $\sigma_{r}$ are assumed to be non-negative constants, while $\theta_{r}(t), t \geq 0$, and $\sigma_{S}(t), t \geq 0$, are supposed to be deterministic functions in time. However, future research might focus on extensions such that $\theta_{r}(t)$ or $\sigma_{S}(t)$ can be stochastic processes. $W_{r}^{\mathbb{Q}}(t)$ and $W_{S}^{\mathbb{Q}}(t)$ represent standard $\mathbb{Q}$-Brownian motions.

\subsection{Mortality Model}

Historical data normally confirm the assumption of an exponential relation between age and one-year death probabilities. For instance, see the plot on the left in Figure 1 illustrating the United Kingdom (U.K.) mortality tables (principal projection, men, 1951-2011) ${ }^{3}$ published by the Office for National Statistics. Furthermore, life expectancy is increasing. If we focus on how the U.K.

3 For the underlying data set see http://www.ons.gov.uk/ons/rel/lifetables/historic-and-projected-mortality-data-fromthe-uk-life-tables/2010-based/rft-qx-principal.xls 
one-year death probability for a fixed age has evolved over time (see the plot on the right in Figure 1), the decline underpins this assertion. But, the second plot of Figure 1 also indicates that there are random fluctuations partially resisting an enduring downturn. To take into account all findings we apply the mortality model described in [6] which is based on [28,29]. We assume an upper limit in age $T^{*}$ (e.g., 125 years) and model under the real-world measure $\mathbb{P}$ the remaining lifetime $\tau^{m}(x)$ of an insured aged $x$ years in the form of the first jump of a Cox process $\left(N^{m}(x+t)\right)_{t \geq 0}$ characterized by a $\mathbb{F}$-measurable intensity $\lambda_{t}^{m}(x+t), t \geq 0$ [30]:

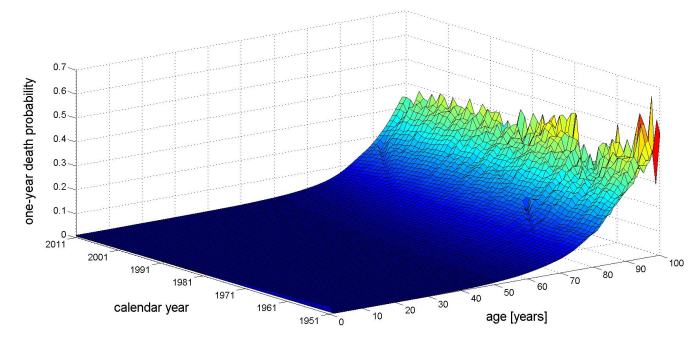

(a)

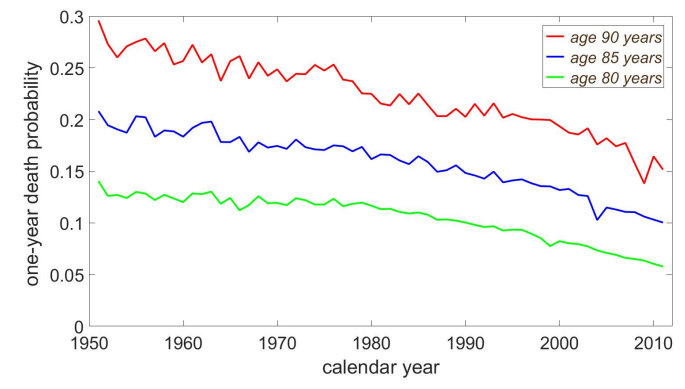

(b)

Figure 1. U.K. Mortality Tables (a) and One-Year Death Probabilities (b) of Men (1951-2011).

$$
\tau^{m}(x):=\min \left(T^{*}-x, \inf \left\{t \geq 0: N^{m}(x+t)>0\right\}\right) .
$$

The index $m$ refers to mortality. Furthermore, we define:

$$
\mathbb{H}^{m}:=\left(\mathcal{H}_{t}^{m}\right)_{t \in\left[0, T^{*}-x\right]}, \mathcal{H}_{t}^{m}:=\sigma\left(\mathbb{1}_{\left\{\tau^{m}(x) \leq u\right\}}: u \leq t\right) .
$$

Hence, $\mathbb{H}^{m}$ captures whether the insured is still alive or has already died up to a certain point in time.

Let the initial mortality intensity $\lambda_{0}^{m}(x+t)$ be given by the static Gompertz model and let an Ornstein-Uhlenbeck process $\xi:=(\xi(t))_{t \geq 0}$ describe the evolution of the $\mathbb{P}$-dynamics of the mortality improvement ratio. Then, we get for the mortality model under $\mathbb{P}$ :

$$
\begin{aligned}
& \lambda_{t}^{m}(x+t)=\lambda_{0}^{m}(x+t) \cdot \xi(t), \\
& \lambda_{0}^{m}(x+t)=\frac{1}{b} \exp \left(\frac{x+t-z}{b}\right), \\
& \mathrm{d} \xi(t)=\kappa(\exp (-\gamma t)-\xi(t)) \mathrm{d} t+\sigma_{\xi} \mathrm{d} W_{\xi}^{\mathbb{P}}(t) .
\end{aligned}
$$

The constants $z, \kappa$ and $\sigma_{\xi}$ are non-negative, $b$ is positive and $\gamma \in \mathbb{R}$. A positive $\gamma$ indicates that people are growing older on average, whereas a negative $\gamma$ implies the opposite. For $\gamma=0$ the mortality improvement ratio is fluctuating close to 1 and hence, there are no trends. In this paper the mortality improvement ratio $\xi$ does not depend on the age $x$ of an insured, since it is supposed to reflect a general improvement in mortality. The correlations between $W_{\tilde{\zeta}}^{\mathbb{P}}(t)$ and the $\mathbb{P}$-Brownian motions of the financial market, are assumed to be zero implying the independence of the insurance and the financial processes. Subsequent measure changes are designed such that this feature is preserved. Using Itô's Lemma we obtain the $\mathbb{P}$-dynamics of the overall mortality intensity:

$$
\mathrm{d} \lambda_{t}^{m}(x+t)=\left(c_{1} \exp \left(c_{2} t\right)-c_{3} \lambda_{t}^{m}(x+t)\right) \mathrm{d} t+c_{4} \exp \left(c_{5} t\right) \mathrm{d} W_{\tilde{\zeta}}^{\mathbb{P}}(t)
$$

with

$$
c_{1}:=\frac{\kappa}{b} \exp \left(\frac{x-z}{b}\right), c_{2}:=\frac{1}{b}-\gamma, c_{3}:=\kappa-\frac{1}{b}, c_{4}:=\frac{\sigma_{\tilde{\xi}}}{b} \exp \left(\frac{x-z}{b}\right), c_{5}:=\frac{1}{b}
$$


Mortality has been modeled under the real-world measure $\mathbb{P}$ so far. However, risk-neutral pricing techniques require probabilities with respect to the risk-neutral measure $\mathbb{Q}$ of the financial market. A lack of transparency, the relatively small number of (variable) annuity providers (supply) compared to the multitude of policyholders (demand) and the informational asymmetry between both parties cause us to reject the assumption of an efficient market for mortality risk [31]. Except for extremely competitive business segments, VA providers should be able to implicitly charge an additional premium for taking longevity and other actuarial risks. On the assumption that all actuarial risks are already taken into account in the form of the mortality tables entering the calibration of the real-world mortality model in (2), the work in [6] assumes that the $\mathbb{P}$ - and $\mathbb{Q}$-survival probabilities coincide. Since this assumption is quite strong, we work with a market price of mortality risk that is permitted to be zero to gain flexibility. Whenever the existence of the market price of mortality risk is difficult to accept as valid, the risk premium can be set to zero to end up in the setting of [6]. If there are good reasons for the existence of a mortality risk premium, which has not yet been covered by the mortality tables themselves, our approach allows its estimation. Furthermore, we are able to analyze how a mortality risk premium affects the $\mathbb{Q}$-survival probabilities (sensitivity tests). To keep our mortality model analytically tractable, in particular, to preserve the independence of the insurance and the financial market, we consider a constant market price of mortality risk $\gamma_{m}$. However, alternative risk premium models like in [32] should be part of future research. In our setting, the Radon-Nikodym density defined by:

$$
\begin{aligned}
\left.\frac{\mathrm{d} \mathbb{Q}}{\mathrm{d} \mathbb{P}}\right|_{\mathcal{F}_{t} \vee \mathcal{H}_{t}^{m}} & =\exp \left(-\gamma_{m} W_{\tilde{\zeta}}^{\mathbb{P}}(t)-\frac{1}{2} \gamma_{m}^{2} t\right), \\
W_{\tilde{\zeta}}^{\mathbb{Q}}(t) & =W_{\tilde{\zeta}}^{\mathbb{P}}(t)+\gamma_{m} t,
\end{aligned}
$$

and the Girsanov theorem provide ${ }^{4}$ :

$$
\mathrm{d} \lambda_{t}^{m}(x+t)=\left(c_{1} \exp \left(c_{2} t\right)-c_{3} \lambda_{t}^{m}(x+t)-c_{4} \gamma_{m} \exp \left(c_{5} t\right)\right) \mathrm{d} t+c_{4} \exp \left(c_{5} t\right) \mathrm{d} W_{\xi}^{\mathbb{Q}}(t) .
$$

\subsection{Surrender Model}

At first, we describe the characteristics of the considered VAs. Thereby, we especially focus on the surrender benefit to model the policyholders' surrender behavior properly. Let $I>0$ be the initial premium which the policyholder has to pay at once at the beginning when entering into the VA contract with maturity $T$. Since $I$ is fully invested in a fund or stock, $A(t):=I \exp (Y(t)), t \in[0, T]$, gives the evolution of the fund account value over time. The contract includes a guaranteed minimum accumulation benefit (GMAB). If $\delta \geq 0$ denotes the preliminary agreed (annual) roll-up rate, $G(t):=I \exp (\delta t)$ specifies how the (implicit) value of the guarantee moves over time. The GMAB is executable at maturity only. This means that only at maturity the policyholder is allowed to choose between the fund account value and the guarantee. In case of early surrender his right of refund is restricted to the current fund account value reduced by the compulsory surrender fee. From the perspective of the policyholders, the charged surrender fee and the forbidden execution of the GMAB option before maturity reduce the incentives for early surrender. For VA providers the combination of both serves as a perfect hedge, since the repayment to the policyholder is less (in the presence of surrender fees) or equal to the fund account value (when the fee is zero). In case of early surrender let $f:[0, T] \rightarrow \mathbb{R}_{0}^{+}$be a non-increasing function of time such that the surrender benefit at $t$ is equal to: $I \exp (Y(t)-f(t))$. If $f$ is properly chosen, we are able to overcome the problem that an insurance

4 In the suggested modeling approach the mortality intensity can become negative with positive probability. This probability can be calculated analytically, see Appendix A.2. However, in practical applications, like for the parameters used in our example (see Section 4 ), this probability is negligible (less than $10^{-5}$ ). 
company could be unable "to fully recover its initial expenses" [8] due to (early) surrender. For all $t \in[0, T]$, let $R(t, T)$ be the annual, continuously compounded long-term interest rate at $t$ for the period $[t, T]$. Let $P(t, T)$ be the price of a default-free zero-coupon bond at time $t$ with maturity $T$. Then, we have that:

$$
R(t, T)=-\frac{1}{T-t} \ln (P(t, T))
$$

Apart from their actuarial characteristics VAs are capital market products. This is why it might happen that policyholders behave similar to investors pursuing a long-term investment strategy. If early surrender takes place at time $t$, the policyholder would be able to reinvest the surrender benefit at $R(t, T)$ for the remaining time to maturity. In this case, the final payoff at maturity would be given by:

$$
I \exp (Y(t)-f(t)+R(t, T)(T-t)) .
$$

In the absence of early surrender, the repayment at maturity is at least $G(T)$, serving as a benchmark for the above long-term strategy. If the stochastic process $D:=(D(t))_{t \geq 0}$ is equal to the logarithm of the ratio of both final payoffs, we have that:

$$
D(t)=Y(t)-f(t)-\delta T+R(t, T)(T-t) .
$$

Similar to the mortality model, let the time $\tau^{s}$ until the early surrender option is exercised be equal to the time until the first jump of a Cox process $\left(N^{s}(t)\right)_{t \geq 0}$ with intensity $\lambda^{s}(t)$ defined by:

$$
\lambda^{s}(t)=\beta \max [\min [D(t), \alpha], 0]+C,
$$

where the constants $\alpha, \beta$ and $C$ are non-negative. The index $s$ in case of $\lambda^{s}$ denotes surrender to distinguish it from the mortality intensity $\lambda^{m}$. The lower limit $C$ covers all policyholders who are willing or obliged to surrender their contracts, for example due to a desire for current consumption or debt, even though it is not rational from the perspective of maximizing policy value. The minimum of $D(t)$ and $\alpha$ allows the construction of an upper limit representing all policyholders never willing to exercise the early surrender option. Due to the chosen construction the surrender intensity cannot become negative. Hence, the surrender probabilities always lie inside the range $[0,1]$. Since the capped (upper limit) and floored (lower limit) linear relation results in a curve having an "s" shape, we will call it s-curve in the sequel. Aside from the analytical tractability, the surrender intensity in (4) offers some advantages regarding the findings derived from the empirical studies.

On the one hand, the higher the long-term interest rate $R(t, T)$, the higher the decision criterion $D(t)$ and hence, the higher the surrender intensity $\lambda^{s}(t)$. This means that an increase in the interest rates results in increased surrender probabilities which is in accordance with the interest-rates hypothesis. On the other hand, an outperforming fund in the form of a large $Y(t)$ also increases $D(t)$ and thus, the surrender intensity. In this case some profit taking by the policyholders is taken into account as well. In practice, surrender fees often decrease with time to make sure that the contract is kept for a while. By contrast, the more time passes the more the guaranteed minimum benefit gains in importance. When the value of the guarantee increases, the early surrender option should be rarely exercised (at least from a rational point of view). As the surrender intensity in (4) is able to incorporate the impact of the surrender fees and the implicit value of the guarantee at maturity, at any point in time it balances the factors increasing the surrender probabilites with the ones doing the opposite such that it is finally driven by the net impact of both trends. 
As before, we define:

$$
\mathbb{H}^{s}:=\left(\mathcal{H}_{t}^{s}\right)_{t \in\left[0, T^{*}-x\right]}, \mathcal{H}_{t}^{s}:=\sigma\left(\mathbb{1}_{\left\{\tau^{s} \leq u\right\}}: u \leq t\right)
$$

indicating whether the early surrender option has been exercised up to a certain point in time.

Because of its definition in (4) the surrender intensity $\lambda^{s}(t), t \geq 0$, is a deterministic function of stochastic inputs from the financial market and so, is $\mathbb{F}$-measurable. Any information on the financial market, mortality and contract surrender up to a certain point in time is covered by the filtration $\mathbb{G}$ with:

$$
\mathbb{G}:=\mathbb{F} \vee \mathbb{H}^{m} \vee \mathbb{H}^{s}
$$

Let $\left(r_{t}, Y_{t}, \lambda_{t}^{m}(x+t)\right)^{\prime}$ be the underlying state process. For any $t \in[0, T]$ Proposition 3.1 in [33] allows us to replace the filtration $\mathcal{G}_{t}$ by the filtration $\mathcal{F}_{t}$ together with indicator functions using $\tau^{\mathcal{S}}$ and $\tau^{m}(x)$ when we determine the present values of $\mathcal{F}_{T}$-measurable final repayments $X_{T} \mathbb{1}_{\{\tau>T\}}$ in the absence of default, i.e., no early death or premature contract surrender. Moreover, Proposition 3.1 in [33] enables this exchange when we price $\mathcal{F}_{u}$-adapted streams of payments $X_{u} \mathbb{1}_{\{\tau>u\}}$ up to default or $\mathcal{F}_{u}$-adapted recovery payments $X_{u}$ at the time of default $\tau=u$. In particular, we have that:

$$
\begin{aligned}
\mathbb{E}_{\mathbb{Q}}\left[\exp \left(-\int_{t}^{T} r(u) \mathrm{d} u\right) \mathbb{1}_{\left\{\tau^{s}>T\right\}} \mathbb{1}_{\left\{\tau^{m}(x)>T\right\}} \mid \mathcal{G}_{t}\right] \\
\quad=\mathbb{1}_{\left\{\tau^{s}>t\right\}} \mathbb{1}_{\left\{\tau^{m}(x)>t\right\}} \mathbb{E}_{\mathbb{Q}}\left[\exp \left(-\int_{t}^{T}\left(r(u)+\lambda_{u}^{m}(x+u)+\lambda^{s}(u)\right) \mathrm{d} u\right) \mid \mathcal{F}_{t}\right] .
\end{aligned}
$$

\section{Products and Approximations}

We begin by specifying the type of VAs to be considered. Unfortunately, there is no unique understanding in the literature of what is meant by guaranteed minimum accumulation benefits (GMABs), surrender benefits (SBs) or guaranteed minimum death benefits (GMDBs). Therefore, we introduce the definitions we are working with, since they are crucial for the later product pricing and the derivation of the closed-form approximation.

\subsection{Product Definitions and Characteristics}

As before, $A(t):=I \exp (Y(t))$ and $G(t):=I \exp (\delta t), t \in[0, T]$, define the (implied) value of the fund and the guarantee. Let $\mathbf{t}:=\left(t_{1}, \ldots, t_{K}\right)^{\prime}$ with $0<t_{1}<\ldots<t_{K}<T$ be the dates on which premature surrender is possible. Since $t_{K}<T$, the early surrender option has to be exercised before maturity, otherwise, the contract will anyway expire at $T$. In this paper the GMAB provides a payoff and hence, financial protection at $T$ only, i.e., the choice between $A(t)$ and $G(t)$ is restricted to $t=T$. This is the reason why $G(t)$ gives for all $t<T$ the implied value of the GMAB. To make sure that a policyholder is always entitled to a single constituent of the overall VA contract the financial protection of the GMAB is valid as long as the insured is still alive (i.e., $\left\{\tau^{m}(x)>T\right\}$ ) and early surrender has not taken place (i.e., $\left\{\tau^{\mathcal{S}}>T\right\}$ ). If we summarize the preceding restrictions, we get for the payoff of the GMAB at time $t \in[0, T]$ :

$$
\operatorname{GMAB}(t, T)=\mathbb{1}_{\{t=T\}} \mathbb{1}_{\left\{\tau^{m}(x)>T\right\}} \mathbb{1}_{\left\{\tau^{s}>T\right\}} \cdot \max [A(T), G(T)]
$$

In case of surrender at time $t_{i}, 1 \leq i \leq K$, the SB is given by: $I \exp \left(Y\left(t_{i}\right)-f\left(t_{i}\right)\right)$ such that there is no guarantee involved. Since the surrender fees are usually stipulated in the VA contract in a determenistic (state-independent) way, the assumption that $f$ is deterministic is not overly restrictive. Assuming that $f$ is decreasing reduces the incentives for early surrender, but it is not required for the subsequent mathematical derivations. Again, to ensure that a policyholder's right of refund is always restricted to a single constituent of the overall VA contract the early surrender option can be exercised only once, if the insured is still alive (i.e., $\left.\left\{\tau^{\mathcal{S}}<\tau^{m}(x)\right\}\right)$ and if this is indicated within the preceding 
notice period (i.e., $\left\{t_{i-1}<\tau^{\mathcal{S}} \leq t_{i}\right\}$ ). Assuming $t_{0}:=0$, the payoff of the SB at time $t_{i}, 1 \leq i \leq K$, is given by:

$$
\mathrm{SB}\left(t_{i}, T\right)=\mathbb{1}_{\left\{t_{i-1}<\tau^{s} \leq t_{i}\right\}} \mathbb{1}_{\left\{\tau^{s}<\tau^{m}(x)\right\}} \cdot I \cdot \exp \left(Y\left(t_{i}\right)-f\left(t_{i}\right)\right) .
$$

The third feature is a GMDB offering protection when the insured dies before contract expiration. Although an insured can die at any point in time (continuous death event), let $\overline{\mathbf{t}}:=\left(\bar{t}_{1}, \ldots, \bar{t}_{N}\right)^{\prime}$ with $0<\bar{t}_{1}<\ldots<\bar{t}_{N}=T$ be the dates the death benefit is paid (discrete repayment dates). In general, the termination dates of the SB and the repayment dates of the GMDB may be different. For simplicity, the roll-up rates of the GMDB and the GMAB are assumed to be equal. In this case, the same roll-up rate is used in the surrender intensity process and in the GMDB guarantee. For the GMDB, there are no fees such that the repayment at $\bar{t}_{i}, 1 \leq i \leq N$, is equal to $\max \left[A\left(\bar{t}_{i}\right), I \exp \left(\delta \bar{t}_{i}\right)\right]$. To avoid double claims of the policyholder, the GMDB provides a payoff before $T$ only once, if the early surrender option has not been exercised so far (i.e., $\left\{\tau^{m}(x)<\tau^{s}\right\}$ ). We define $\bar{t}_{0}:=0$. If the insured dies within the period $\left\{\bar{t}_{i-1}<\tau^{m}(x) \leq \bar{t}_{i}\right\}$, the payoff of the GMDB at repayment date $\bar{t}_{i}, 1 \leq i \leq N$ is given by:

$$
\operatorname{GMDB}\left(\bar{t}_{i}, T\right)=\mathbb{1}_{\left\{\bar{t}_{i-1}<\tau^{m}(x) \leq \bar{t}_{i}\right\}} \mathbb{1}_{\left\{\tau^{m}(x)<\tau^{s}\right\}} \cdot \max \left[A\left(\bar{t}_{i}\right), I \exp \left(\delta \bar{t}_{i}\right)\right] .
$$

The above GMABs, SBs and GMDBs are designed such that a policyholder is not able to have two claims at the same time. Therefore, the payoff at time $t \in[0, T]$ of the overall VA is given by:

$$
\mathrm{VA}(t, T)=\operatorname{GMAB}(t, T)+\mathrm{SB}(t, T)+\operatorname{GMDB}(t, T)
$$

\subsection{Product Pricing and Required Approximations}

We assume an arbitrage-free market, and so, the price of the overall VA at time $t=0$, i.e., $\mathrm{p}^{\mathrm{VA}}(0)$, has to be equal to the sum of the corresponding prices of its constituents.

$$
\mathrm{p}^{\mathrm{VA}}(0)=\mathrm{p}^{\mathrm{GMAB}}(0)+\mathrm{p}^{\mathrm{SB}}(0)+\mathrm{p}^{\mathrm{GMDB}}(0) .
$$

For any point in time (5)-(8) provide the payoffs of the overall VA contract and its components. To end up with the prices in (9) all payments received during the contract period $[0, T]$ have to be discounted.

By virtue of risk-neutral pricing, the present value of the GMAB (conditioning on $\mathcal{G}_{0}$ has been omitted as no further information is provided) is given by:

$$
\begin{aligned}
p^{\operatorname{GMAB}}(0) & =\mathbb{E}_{\mathbb{Q}}\left[\exp \left(-\int_{0}^{T} r(u) \mathrm{d} u\right) \operatorname{GMAB}(T, T)\right] \\
& =\mathbb{Q}\left(\tau^{m}(x)>T\right) \cdot G(T) \cdot P(0, T) \cdot\left(E_{1}\left[\lambda^{s}\right]+E_{2}\left[\lambda^{s}\right]\right),
\end{aligned}
$$

with

$$
\begin{array}{ll}
\mathbb{Q}\left(\tau^{m}(x)>T\right) & =\mathbb{E}_{\mathbb{Q}}\left[\exp \left(-\int_{0}^{T} \lambda_{u}^{m}(x+u) \mathrm{d} u\right)\right], \\
P(0, T) & =\mathbb{E}_{\mathbb{Q}}\left[\exp \left(-\int_{t}^{T} r(u) \mathrm{d} u\right)\right], \\
E_{1}\left[\lambda^{s}\right] & =\mathbb{E}_{\mathbb{Q}^{T}}\left[\exp \left(-\int_{0}^{t_{K}} \lambda^{s}(u) \mathrm{d} u\right)\right], \\
E_{2}\left[\lambda^{s}\right] & =\mathbb{E}_{\mathbb{Q}^{T}}\left[\exp \left(-\int_{0}^{t_{K}} \lambda^{s}(u) \mathrm{d} u\right)(\exp (Y(T)-\delta T)-1)^{+}\right] .
\end{array}
$$

Using (10) the price of the GMAB can be converted into a product of already known factors, except the one in the form of $\left(E_{1}\left[\lambda^{s}\right]+E_{2}\left[\lambda^{s}\right]\right)$ which will be explained in detail later on. The first factor reflects the risk-neutral probability that the insured does not die early. The second factor displays 
the guaranteed, final payoff. The third factor is given by the price of a default-free zero-coupon bond with maturity $T$. Hence, the product of factors 2 and 3 provides the present value of the guarantee of the GMAB. The expressions for $E_{1}\left[\lambda^{s}\right]$ and $E_{2}\left[\lambda^{s}\right]$ employ the forward measure $\mathbb{Q}^{T}$ defined through the Radon-Nikodym derivative:

$$
\left.\frac{d \mathbb{Q}^{T}}{d \mathbb{Q}}\right|_{\mathcal{G}_{0}}=\frac{P(T, T) / P(0, T)}{B(T) / B(0)}
$$

with

$$
B(t):=\exp \left(\int_{0}^{t} r(u) \mathrm{d} u\right), B(0)=1 .
$$

$E_{1}\left[\lambda^{s}\right]$ represents the forward probability that no surrender takes place before $T$ (the last possible termination date is $t_{K}$ ), i.e., that the early surrender option is never exercised. Therefore, the first summand in (10) can be interpreted as the surrender and mortality risk adjusted present value of the guarantee $G(T)$. Since $E_{2}\left[\lambda^{s}\right]$ shows similarities to a European call option, the second summand in (10) can be considered as the surrender and mortality risk adjusted value of a European call option with maturity $T$ written on the fund account value with strike $G(T)$. Hence, it covers the surplus of the fund compared to the guarantee, if the contract is kept in force until maturity.

Owing to their nonlinearity in $\lambda^{s}$, closed-form expressions for $E_{1}\left[\lambda^{s}\right]$ and $E_{2}\left[\lambda^{s}\right]$ are not available, and we resort to analytical approximations. If we replace $\lambda^{s}$ by its definition in (4) and apply rectangular integration, we get for the first term:

$$
\begin{aligned}
E_{1}\left[\lambda^{s}\right] & =\exp \left(-C \cdot t_{K}\right) \cdot \mathbb{E}_{\mathbb{Q}^{T}}\left[\exp \left(-\beta \int_{0}^{t_{K}} \max [\min [D(u), \alpha], 0] \mathrm{d} u\right)\right] \\
& \approx \exp \left(-C \cdot t_{K}\right) \cdot \mathbb{E}_{\mathbb{Q}^{T}}\left[\exp \left(-\beta \sum_{i=1}^{K} \Delta t_{i} \max \left[\min \left[D\left(t_{i}\right), \alpha\right], 0\right]\right)\right]
\end{aligned}
$$

with $\Delta t_{i}:=t_{i}-t_{i-1}, 1 \leq i \leq K$. Here, we would like to highlight that the integral is not approximated through an arbitrarily fine grid decreasing the approximation error at the expense of the enhanced numerical effort. The grid points are defined by t. A rational policyholder exactly exercises the surrender option at one of the termination dates, but not in the meantime, as, e.g., the fund performance may dramatically worsen until his surrender decision takes effect. Therefore, the changes in $\lambda^{s}$ between $t_{i-1}$ and $t_{i}$ are of minor importance so that the surrender intensity at a termination date multiplied by the time period from the last observation serves as a proper approximation for the integrated surrender intensity.

Next, we use that it holds for all $1 \leq i \leq K$ :

$$
\max \left[\min \left[D\left(t_{i}\right), \alpha\right], 0\right]=D\left(t_{i}\right) \cdot \mathbb{1}_{\left\{0<D\left(t_{i}\right)<\alpha\right\}}+\alpha \cdot \mathbb{1}_{\left\{D\left(t_{i}\right) \geq \alpha\right\}} \cdot
$$

Thereafter, we take into account that the exponential function represents an absolutely convergent power series which implies that a changed order of summation preserves the limit. If $x_{1}, \ldots, x_{n}$ are arbitrary real numbers, the reordering of the exponential function we are working with is given by:

$$
\begin{aligned}
\exp \left(\sum_{i=1}^{n} x_{i}\right) & =\sum_{k=0}^{\infty} \frac{\left(\sum_{i=1}^{n} x_{i}\right)^{k}}{k !} \\
& =1+\sum_{i=1}^{n}\left(\exp \left(x_{i}\right)-1\right)+\sum_{\substack{i, j=1 \\
i<j}}^{n} x_{i} x_{j}+\mathbf{O}(\text { mixed terms of order } \geq 3),
\end{aligned}
$$


where $\mathbf{O}(\cdot)$ denotes the Landau notation. To illustrate the quality of this approximation Figure 2 displays the approximation error for $^{5} n=2,-0.15<x_{i}<0, \quad i=1,2$. We can observe that in all cases the approximation error is below $3 \cdot 10^{-3}$.

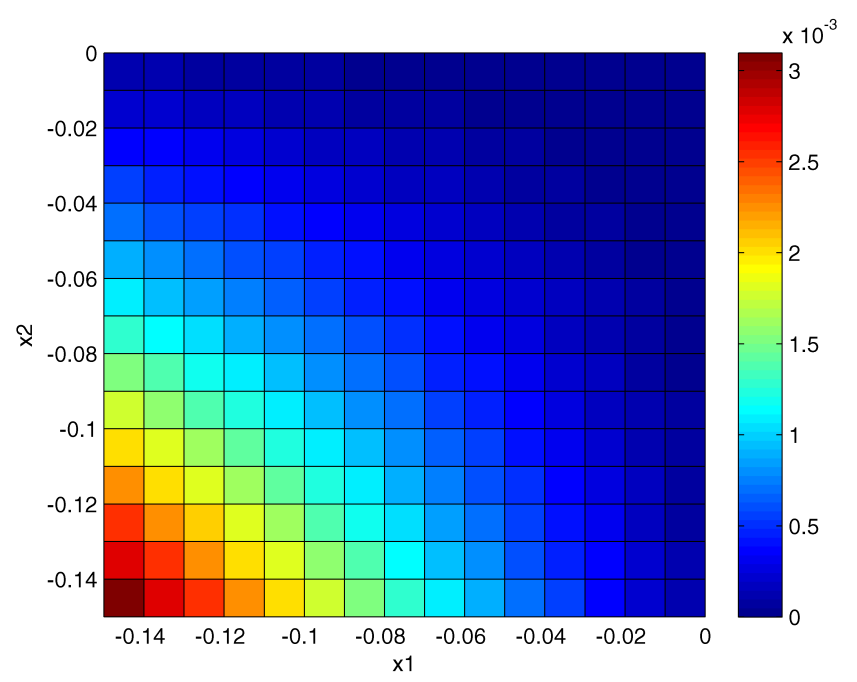

Figure 2. Approximation Error for Equation (13).

Finally, we focus on a reduced number of summands of the reordered power series. For this purpose, we omit all mixed terms of order $\geq 3$ and some of the mixed terms of order 2 and get:

$$
E_{1}\left[\lambda^{s}\right] \approx \exp \left(-C \cdot t_{K}\right) \cdot\left(S_{11}^{\mathbb{Q}^{T}}(D, \alpha, \beta, \mathbf{t})+S_{12}^{\mathbb{Q}^{T}}(D, \alpha, \beta, \mathbf{t})\right),
$$

with:

$$
\begin{aligned}
S_{11}^{\mathbb{Q}^{T}}(D, \alpha, \beta, \mathbf{t}):= & 1+\sum_{i=1}^{K} \mathbb{E}_{\mathbb{Q}^{T}}\left[\left(\exp \left(-\beta \Delta t_{i} D\left(t_{i}\right)\right)-1\right) \mathbb{1}_{\left\{0<D\left(t_{i}\right)<\alpha\right\}}\right] \\
& +\sum_{i=1}^{K} \mathbb{E}_{\mathbb{Q}^{T}}\left[\left(\exp \left(-\beta \Delta t_{i} \alpha\right)-1\right) \mathbb{1}_{\left\{D\left(t_{i}\right) \geq \alpha\right\}}\right], \\
S_{12}^{\mathbb{Q}^{T}}(D, \alpha, \beta, \mathbf{t}):= & \sum_{\substack{i, j=1 \\
i<j}}^{K} \beta^{2} \Delta t_{i} \Delta t_{j} \mathbb{E}_{\mathbb{Q}^{T}}\left[D\left(t_{i}\right) D\left(t_{j}\right) \mathbb{1}_{\left\{0<D\left(t_{i}\right)<\alpha\right\}} \mathbb{1}_{\left\{0<D\left(t_{j}\right)<\alpha\right\}}\right] \\
+ & \sum_{\substack{i, j=1 \\
i<j}}^{K} \beta^{2} \alpha^{2} \Delta t_{i} \Delta t_{j} \mathbb{Q}^{T}\left(D\left(t_{i}\right) \geq \alpha, D\left(t_{j}\right) \geq \alpha\right) .
\end{aligned}
$$

For the ease of exposition, we define $\tilde{Y}(t):=Y(t)-\delta t$ for all $t \in[0, T]$. If we apply the same methods as before, but omit all mixed terms of order $\geq 2$, we get for $E_{2}\left[\lambda^{s}\right]$ :

$$
E_{2}\left[\lambda^{S}\right] \approx \exp \left(-C \cdot t_{K}\right) \cdot S_{2}^{\mathbb{Q}^{T}}(D, \tilde{Y}, \alpha, \beta, \mathbf{t}),
$$

with

$$
\begin{aligned}
S_{2}^{\mathbb{Q}^{T}}(D, \tilde{Y}, \alpha, \beta, \mathbf{t}): & =\mathbb{E}_{\mathbb{Q}^{T}}\left[\exp (\tilde{Y}(T)) \mathbb{1}_{\{\tilde{Y}(T) \geq 0\}}\right]-\mathbb{Q}^{T}(\tilde{Y}(T) \geq 0) \\
& +\sum_{i=1}^{K} \mathbb{E}_{\mathbb{Q}^{T}}\left[\left(\exp \left(-\beta \Delta t_{i} D\left(t_{i}\right)\right)-1\right)(\exp (\tilde{Y}(T))-1) \mathbb{1}_{\left\{0<D\left(t_{i}\right)<\alpha\right\}} \mathbb{1}_{\{\tilde{Y}(T) \geq 0\}}\right] \\
& +\sum_{i=1}^{K} \mathbb{E}_{\mathbb{Q}^{T}}\left[\left(\exp \left(-\beta \alpha \Delta t_{i}\right)-1\right)(\exp (\tilde{Y}(T))-1) \mathbb{1}_{\left\{D\left(t_{i}\right) \geq \alpha\right\}} \mathbb{1}_{\{\tilde{Y}(T) \geq 0\}}\right] .
\end{aligned}
$$

\footnotetext{
5 This will be the range for the corresponding expressions in our numerical case studies.
} 
If we define for all $1 \leq i \leq K$ :

$$
\begin{aligned}
& h_{1}\left(\beta, D, t_{i}\right):=-\beta \Delta t_{i} \mathbb{E}_{\mathbb{Q}^{T}}\left[D\left(t_{i}\right)\right]+\frac{1}{2} \beta^{2} \Delta t_{i}^{2} \operatorname{Var}_{\mathbb{Q}^{T}}\left[D\left(t_{i}\right)\right], \\
& h_{2}\left(\alpha, \beta, D, t_{i}\right):=\frac{\mathbb{E}_{\mathbb{Q}^{T}}\left[D\left(t_{i}\right)\right]-\alpha}{\sqrt{\operatorname{Var}_{\mathbb{Q}^{T}}\left[D\left(t_{i}\right)\right]}}-\beta \Delta t_{i} \sqrt{\operatorname{Var}_{\mathbb{Q}^{T}}\left[D\left(t_{i}\right)\right]},
\end{aligned}
$$

we get for $S_{11}^{\mathbb{Q}^{T}}(D, \alpha, \beta, \mathbf{t})$ :

$$
\begin{aligned}
S_{11}^{\mathbb{Q}^{T}}(D, \alpha, \beta, \mathbf{t})=1 & +\sum_{i=1}^{K} \exp \left(h_{1}\left(\beta, D, t_{i}\right)\right) \Phi\left(h_{2}\left(0, \beta, D, t_{i}\right)\right)-\sum_{i=1}^{K} \exp \left(h_{1}\left(\beta, D, t_{i}\right)\right) \Phi\left(h_{2}\left(\alpha, \beta, D, t_{i}\right)\right) \\
& +\sum_{i=1}^{K} \exp \left(-\beta \alpha \Delta t_{i}\right) \Phi\left(h_{2}\left(\alpha, 0, D, t_{i}\right)\right)-\sum_{i=1}^{K} \Phi\left(h_{2}\left(0,0, D, t_{i}\right)\right),
\end{aligned}
$$

where $\Phi(\cdot)$ denotes the cumulative distribution function (CDF) of the standard Gaussian $\mathcal{N}(0,1)$. The detailed proof is shown in Appendix C.1.

For all $i, j \in\{1, \ldots, K\}$ with $i<j$ we define:

$$
\begin{aligned}
& \mu(i, j):=\left(\begin{array}{cc}
\mathbb{E}_{\mathbb{Q}^{T}}\left[D\left(t_{i}\right)\right] \\
\mathbb{E}_{\mathbb{Q}^{T}}\left[D\left(t_{j}\right)\right]
\end{array}\right), \\
& \Sigma(i, j):=\left(\begin{array}{cc}
\operatorname{Var}_{\mathbb{Q}^{T}}\left[D\left(t_{i}\right)\right] & \operatorname{Cov}_{\mathbb{Q}^{T}}\left[D\left(t_{i}\right), D\left(t_{j}\right)\right] \\
\operatorname{Cov}_{\mathbb{Q}^{T}}\left[D\left(t_{i}\right), D\left(t_{j}\right)\right] & \operatorname{Var}_{\mathbb{Q}^{T}}\left[D\left(t_{j}\right)\right]
\end{array}\right) .
\end{aligned}
$$

After the indicator set in (16) has been split, we have:

$$
\begin{aligned}
S_{12}^{\mathbb{Q}^{T}}(D, \alpha, \beta, \mathbf{t}): & =\sum_{\substack{i, j=1 \\
i<j}}^{K} \beta^{2} \Delta t_{i} \Delta t_{j} \mathbb{E}_{\mathbb{Q}^{T}}\left[D\left(t_{i}\right) D\left(t_{j}\right) \mathbb{1}_{\left\{0<D\left(t_{i}\right), 0<D\left(t_{j}\right)\right\}}\right] \\
& -\sum_{\substack{i, j=1 \\
i<j}}^{K} \beta^{2} \Delta t_{i} \Delta t_{j} \mathbb{E}_{\mathbb{Q}^{T}}\left[D\left(t_{i}\right) D\left(t_{j}\right) \mathbb{1}_{\left\{0<D\left(t_{i}\right), \alpha \leq D\left(t_{j}\right)\right\}}\right] \\
& -\sum_{\substack{i, j=1 \\
i<j}}^{K} \beta^{2} \Delta t_{i} \Delta t_{j} \mathbb{E}_{\mathbb{Q}^{T}}\left[D\left(t_{i}\right) D\left(t_{j}\right) \mathbb{1}_{\left\{\alpha \leq D\left(t_{i}\right), 0<D\left(t_{j}\right)\right\}}\right] \\
& +\sum_{\substack{i, j=1 \\
i<j}}^{K} \beta^{2} \Delta t_{i} \Delta t_{j} \mathbb{E}_{\mathbb{Q}^{T}}\left[D\left(t_{i}\right) D\left(t_{j}\right) \mathbb{1}_{\left\{\alpha \leq D\left(t_{i}\right), \alpha \leq D\left(t_{j}\right)\right\}}\right] \\
& +\sum_{\substack{i, j=1 \\
i<j}}^{K} \alpha^{2} \beta^{2} \Delta t_{i} \Delta t_{j} M\left((0,0)^{\prime}, \mu(i, j), \Sigma(i, j),(\alpha, \alpha)^{\prime}, I_{2}\right) .
\end{aligned}
$$

where $I_{2}$ is the two-dimensional identity matrix. The expectations of the product of the truncated random variables $D\left(t_{i}\right)$ and $D\left(t_{j}\right)$ are provided in Appendix B.4. The expression $M(\cdot)$ is explained in Appendix B.2.

For all $1 \leq i \leq K$ we set:

$$
\begin{aligned}
& \mu(i):=\left(\begin{array}{c}
\mathbb{E}_{\mathbb{Q}^{T}}\left[D\left(t_{i}\right)\right] \\
\mathbb{E}_{\mathbb{Q}^{T}}[\tilde{Y}(T)]
\end{array}\right), \\
& \Sigma(i):=\left(\begin{array}{cc}
\operatorname{Var}_{\mathbb{Q}^{T}}\left[D\left(t_{i}\right)\right] & \operatorname{Cov}_{\mathbb{Q}^{T}}\left[D\left(t_{i}\right), \tilde{Y}(T)\right] \\
\operatorname{Cov}_{\mathbb{Q}^{T}}\left[\tilde{Y}(T), D\left(t_{i}\right)\right] & \operatorname{Var}_{\mathbb{Q}^{T}}[\tilde{Y}(T)]
\end{array}\right) .
\end{aligned}
$$


Then, $S_{2}^{\mathbb{Q}^{T}}(D, \tilde{Y}, \alpha, \beta, \mathbf{t})$ can be converted into:

$$
\begin{aligned}
S_{2}^{\mathbb{Q}^{T}}(D, \tilde{Y}, \alpha, \beta, \mathbf{t}) & =\exp \left(\mathbb{E}_{\mathbb{Q}^{T}}[\tilde{Y}(T)]+\frac{1}{2} \operatorname{Var}_{\mathbb{Q}^{T}}[\tilde{Y}(T)]\right) \Phi\left(\frac{\left.\mathbb{E}_{\mathbb{Q}^{T}[\tilde{Y}(T)]+\operatorname{Var}_{\mathbb{Q}^{T}}[\tilde{Y}(T)]}^{\sqrt{\operatorname{Var}_{\mathbb{Q}^{T}}[\tilde{Y}(T)]}}\right)}{}+\sum_{i=1}^{K} M\left(\left(-\beta \Delta t_{i}, 1\right)^{\prime}, \mu(i), \Sigma(i),(0,0)^{\prime}, I_{2}\right)\right. \\
& -\sum_{i=1}^{K} M\left(\left(-\beta \Delta t_{i}, 1\right)^{\prime}, \mu(i), \Sigma(i),(\alpha, 0)^{\prime}, I_{2}\right) \\
& -\sum_{i=1}^{K} M\left(\left(-\beta \Delta t_{i}, 0\right)^{\prime}, \mu(i), \Sigma(i),(0,0)^{\prime}, I_{2}\right) \\
& +\sum_{i=1}^{K} M\left(\left(-\beta \Delta t_{i}, 0\right)^{\prime}, \mu(i), \Sigma(i),(\alpha, 0)^{\prime}, I_{2}\right) \\
& -\sum_{i=1}^{K} M\left((0,1)^{\prime}, \mu(i), \Sigma(i),(0,0)^{\prime}, I_{2}\right) \\
& +\sum_{i=1}^{K} \exp \left(-\beta \alpha \Delta t_{i}\right) M\left((0,1)^{\prime}, \mu(i), \Sigma(i),(\alpha, 0)^{\prime}, I_{2}\right) \\
& +\sum_{i=1}^{K} M\left((0,0)^{\prime}, \mu(i), \Sigma(i),(0,0)^{\prime}, I_{2}\right) \\
& -\sum_{i=1}^{K} \exp \left(-\beta \alpha \Delta t_{i}\right) M\left((0,0)^{\prime}, \mu(i), \Sigma(i),(\alpha, 0)^{\prime}, I_{2}\right)-\Phi\left(\frac{\mathbb{E}_{\mathbb{Q}^{T}}[\tilde{Y}(T)]}{\sqrt{\operatorname{Var}_{\mathbb{Q}^{T}}[\tilde{Y}(T)]}}\right) .
\end{aligned}
$$

For the expression $M(\cdot)$ see Appendix B.2. A detailed proof is shown in Appendix C.2.

Finally, $S_{11}^{\mathbb{Q}^{T}}(D, \alpha, \beta, \mathbf{t}), S_{12}^{\mathbb{Q}^{T}}(D, \alpha, \beta, \mathbf{t})$ and $S_{2}^{\mathbb{Q}^{T}}(D, \tilde{Y}, \alpha, \beta, \mathbf{t})$ only require the moments and covariances in Appendix A.1 and thus, GMABs can be priced based on closed-form approximations. Besides the CDF of the standard Gaussian and the exponential function, only sums, products and quotients are involved. Hence, there are no integrals left causing further numerical effort. The number of summands depends on $\mathbf{t}$ implying that the decomposition of the grid may be orientated towards practical needs. For $T=15$ and a semiannual surrender option we have 29 termination dates, which is easy to handle. The main drawback of our approach is the effort we have to put into its implementation. If we price GMABs using Monte Carlo Simulation (MC), the time and effort we have to spend on implementation may be smaller than in our case. However, to ensure that the provided prices are reliable, many trajectories have to be generated taking time and computational power. For instance, in Case 1 of Table 7 we simulate 500,000 paths for this purpose. By contrast, as soon as the approximation is implemented it takes significantly less time to get comparable prices. Since the implementation has to be done only once at the beginning, whereas performing the MCs can happen serveral times each day, our approach can be justified.

For the present value of the surrender benefit we have that:

$$
\begin{aligned}
\mathrm{p}^{\mathrm{SB}}(0) & =\mathbb{E}_{\mathbb{Q}}\left[\exp \left(-\int_{0}^{\tau^{s}} r(u) \mathrm{d} u\right) \mathrm{SB}\left(\tau^{s}, T\right)\right] \\
& =\sum_{i=1}^{K} \mathbb{E}_{\mathbb{Q}}\left[I \exp \left(-\int_{0}^{t_{i}} r(u) \mathrm{d} u+Y\left(t_{i}\right)-f\left(t_{i}\right)\right) \mathbb{1}_{\left\{t_{i}<\tau^{m}(x)\right\}} \mathbb{1}_{\left\{t_{i-1}<\tau^{s} \leq t_{i}\right\}}\right] \\
& =I \sum_{i=1}^{K} \exp \left(-f\left(t_{i}\right)\right) \cdot \mathbb{Q}\left(\tau^{m}(x)>t_{i}\right) \cdot \mathbb{E}_{\mathbb{Q}}\left[\exp \left(-\int_{0}^{t_{i}} r(u) \mathrm{d} u+Y\left(t_{i}\right)\right) \mathbb{1}_{\left\{t_{i-1}<\tau^{s} \leq t_{i}\right\}}\right], \\
& =I \sum_{i=1}^{K} \exp \left(-f\left(t_{i}\right)\right) \cdot \mathbb{Q}\left(\tau^{m}(x)>t_{i}\right) \cdot \mathbb{Q}^{S\left(t_{i}\right)}\left(t_{i-1}<\tau^{s} \leq t_{i}\right) .
\end{aligned}
$$

In the second line we use the fact that the surrender benefit is only repaid at certain points in time while the third line arises from the independence of the financial market and mortality. Eventually, 
for all periods $\left[0, t_{i}\right]$ with $1 \leq i \leq K$ we replace the risk-neutral measure $\mathbb{Q}$ with the respective equity measure $\mathbb{Q}^{S\left(t_{i}\right)}$, whose Radon-Nikodym derivative obeys for $t<t_{i}$ :

$$
\left.\frac{d \mathbb{Q}^{S\left(t_{i}\right)}}{d \mathbb{Q}}\right|_{\mathcal{G}_{t}}=\frac{S\left(t_{i}\right) / S(t)}{B\left(t_{i}\right) / B(t)}
$$

Therefore, at any termination date $t_{i}$ the surrender benefit in (25) is equal to the present value of the expected fund performance reduced by the prevailing surrender fee, if the insured is still alive $\left(\tau^{m}(x)>t_{i}\right)$ and if the surrender option has been exercised within the preceding surrender period $\left(t_{i-1}<\tau^{\mathcal{S}} \leq t_{i}\right)$. Again, we neglect all mixed terms of order $\geq 2$, and so, obtain for the probability that no early surrender occurs before time $t_{i}, \mathbb{Q}^{S\left(t_{i}\right)}\left(\tau^{\mathcal{S}}>t_{i}\right)$, for $\tilde{\mathbf{t}}_{\mathbf{i}}:=\left\{t_{1}, \ldots, t_{i}\right\}$ :

$$
\begin{aligned}
\mathbb{Q}^{S\left(t_{i}\right)}\left(\tau^{S}>t_{i}\right) & =\mathbb{E}_{\mathbb{Q}^{S\left(t_{i}\right)}}\left[\exp \left(-\int_{0}^{t_{i}} \lambda^{s}(u) \mathrm{d} u\right)\right] \\
& \approx \exp \left(-C \cdot t_{i}\right) \cdot S_{11}^{\mathbb{Q}^{S\left(t_{i}\right)}}\left(D, \alpha, \beta, \tilde{\mathbf{t}}_{\mathbf{i}}\right) .
\end{aligned}
$$

The moments of the decision criterion $D$ under the equity measure $\mathbb{Q}^{S\left(t_{i}\right)}$ are provided in Appendix A.1.

Eventually, we get for the present value of the GMDB:

$$
\begin{aligned}
& \mathrm{p}^{\mathrm{GMDB}}(0)=\mathbb{E}_{\mathbb{Q}}\left[\exp \left(-\int_{0}^{\tau^{m}(x)} r(u) \mathrm{d} u\right) \operatorname{GMDB}\left(\tau^{m}(x), T\right)\right] \\
& =\sum_{i=1}^{N} \mathbb{E}_{\mathbb{Q}}\left[\exp \left(-\int_{0}^{\bar{t}_{i}} r(u) \mathrm{d} u\right) \mathbb{1}_{\left\{\bar{t}_{i}<\tau^{s}\right\}} \cdot \max \left[A\left(\bar{t}_{i}\right), I \exp \left(\delta \bar{t}_{i}\right)\right]\right] \mathbb{Q}\left(\bar{t}_{i-1}<\tau^{m}(x) \leq \bar{t}_{i}\right) \\
& =\sum_{i=1}^{N} \frac{\operatorname{GMAB}\left(0, \bar{t}_{i}\right) \cdot \mathbb{Q}\left(\bar{t}_{i-1}<\tau^{m}(x) \leq \bar{t}_{i}\right)}{\mathbb{Q}\left(\tau^{m}(x)>t_{i}\right)} .
\end{aligned}
$$

Using (28) a GMDB serves as a portfolio consisting of $N$ (the number of repayment dates of the death benefit) mortality adjusted GMAB contracts with maturities $\overline{\mathfrak{t}}$. If we use the closed-form approximation for the GMAB in (10), we get rid of the denominator $\mathbb{Q}\left(\tau^{m}(x)>t_{i}\right)$. As soon as a closed-form solution for GMABs is derived, (28) provides an analytic solution for GMDBs. Because of (11) there are no unknown terms in (28). Another important consequence of (28) is the flexibility inherent in the presented approach, as it supports the reuse of existing expressions and hence, allows for encapsulation when it comes to its implementation.

\section{Model Calibration}

In the sequel, we will calibrate the stochastic models in Section 2 using actively traded financial products and historical mortality tables. Due to a lack of real data, the calibration of the surrender model relies on hypothetical data. However, those insurance companies that have access to sufficient surrender data can easily perform all steps with their inputs. The idea behind this section is to show how the underlying models can be properly calibrated. We do not claim that this is the one and only way. When actively traded financial products enter the calibration, for instance, they can be used when corresponding trading strategies are set up or hedged. Hence, a link between the estimated models and future actions regarding risk and asset management can be established.

\subsection{Financial Market Model}

For any $0 \leq t \leq T$ let $P(t, T)$ be the value of a default-free zero-coupon bond at time $t$ with maturity $T$ in (11). Then, it holds in [34] that:

$$
P(t, T)=\exp \left(A_{r}^{\mathbb{Q}}(t, T)+B_{r}^{\mathbb{Q}}(t, T) r(t)\right)
$$


with

$$
\begin{aligned}
A_{r}^{\mathbb{Q}}(t, T) & =\int_{t}^{T} \theta_{r}(s) B_{r}^{\mathbb{Q}}(s, T) \mathrm{d} s+\frac{1}{2} \sigma_{r}^{2} \int_{t}^{T} B_{r}^{\mathbb{Q}}(s, T)^{2} \mathrm{~d} s, \\
B_{r}^{\mathbb{Q}}(t, T) & =\frac{1}{a_{r}}\left[\exp \left(a_{r}(t-T)\right)-1\right] .
\end{aligned}
$$

Let $f(0, T)$ denote the instantaneous forward rate of $P(0, T)$ at time $t=0$, i.e.,

$$
f(0, T)=-\frac{\partial}{\partial T} \ln (P(0, T)) .
$$

By taking the partial derivative with respect to $T$ (two times) of the logarithm of both sides of (29) the Leibniz's rule in [35] provides for the function $\theta_{r}(t), t \geq 0$ :

$$
\theta_{r}(t)=\frac{\partial}{\partial t} f(0, t)+a_{r} f(0, t)+\frac{\sigma_{r}^{2}}{2 a_{r}}\left[1-\exp \left(-2 a_{r} t\right)\right] .
$$

Inserting this in (29) we get:

$$
\begin{aligned}
A_{r}^{\mathbb{Q}}(t, T) & =\frac{\sigma_{r}^{2}}{4 a_{r}^{3}}\left[\exp \left(-2 a_{r} T\right)-1-\exp \left(2 a_{r}(t-T)\right)+\exp \left(-2 a_{r} t\right)\right]+\ln \left(\frac{P(0, T)}{P(0, t)}\right) \\
& +\frac{\sigma_{r}^{2}}{2 a_{r}^{3}}\left[\exp \left(a_{r}(t-T)\right)-\exp \left(-a_{r}(t+T)\right)\right]+f(0, t) \frac{1-\exp \left(a_{r}(t-T)\right)}{a_{r}} .
\end{aligned}
$$

The calibration of $\theta_{r}(t), t \geq 0$, relies on the zero-coupon bond prices in Figure 3 which are derived from Reuters quotes of 6 month EURIBOR interest-rate swaps (downloaded on 16 January 2013). For missing interest-rate swaps with maturity less than one year suitable EURIBOR spot rates are chosen. We follow Sections 5.5.1-5.5.4 in [36] to obtain the discount curve in Figure 3. As Reuters provides quotes of caps and floors written on the 6 month EURIBOR, for the estimation of $a_{r}$ and $\sigma_{r}$ the mid price at-the-money (ATM) Black volatilities of Table 1 are used. First, these ATM Black volatilities have to be converted into market prices, see Sections 5.8.2-5.8.3 in [36]. Then, the prices of caps and floors are related to portfolios consisting of options written on default-free zero-coupon bonds as in Sections 5.6.1-5.6.2 in [36]. Finally, we minimize the squared difference between the theoretical cap prices provided by the Hull-White model as a function of the unknown parameters $a_{r}$ and $\sigma_{r}$ and the market prices derived from the Black volatilities under the conditions $a_{r} \geq 0$ and $\sigma_{r} \geq 0$. The resulting optimal parameters are given in Table 2.

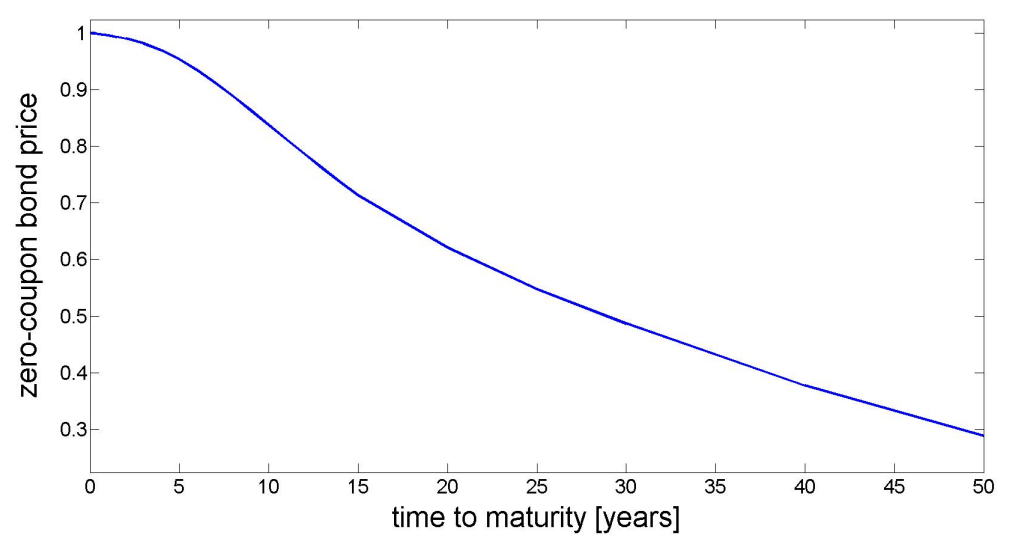

Figure 3. Discount Curve on 16 January 2013. 
Table 1. 6 Month EURIBOR Mid Price ATM Volatilities [\%].

\begin{tabular}{cccccccccc}
\hline Maturity & $\mathbf{2 Y}$ & $\mathbf{3 Y}$ & $\mathbf{4 Y}$ & $\mathbf{5 Y}$ & $\mathbf{6 Y}$ & $\mathbf{7 Y}$ & $\mathbf{8 Y}$ & $\mathbf{9 Y}$ & $\mathbf{1 0 Y}$ \\
\hline Volatility & 99.7 & 66.1 & 61.2 & 55.4 & 50.0 & 45.3 & 41.6 & 38.5 & 36.1 \\
\hline
\end{tabular}

Based on (1) let Option $(t, T, S, K, \psi)$ be the value at time $t<T$ of a European call $(\psi=1)$ or put $(\psi=-1)$ option with strike $K$, maturity $T$ and underlying $S$. Then, it holds:

$$
\text { Option }(t, T, S, K, \psi)=\psi S(t) \Phi\left(\psi h_{S}\right)-\psi K P(t, T) \Phi\left(\psi h_{T}\right),
$$

with

$$
\begin{aligned}
& h_{S}:=\frac{\ln \left(\frac{S(t)}{K}\right)-Y(t)+\mathbb{E}_{\mathbb{Q}^{S(T)}}\left[Y(T) \mid \mathcal{G}_{t}\right]}{\sqrt{\operatorname{Var}_{\mathbb{Q}^{S(T)}}\left[Y(T) \mid \mathcal{G}_{t}\right]}}, \\
& h_{T}:=\frac{\ln \left(\frac{S(t)}{K}\right)-Y(t)+\mathbb{E}_{\mathbb{Q}^{T}}\left[Y(T) \mid \mathcal{G}_{t}\right]}{\sqrt{\operatorname{Var}_{\mathbb{Q}^{T}}\left[Y(T) \mid \mathcal{G}_{t}\right]}} .
\end{aligned}
$$

The index $\mathbb{Q}^{T}$ denotes the forward measure in (12), whereas $\mathbb{Q}^{S(T)}$ denotes the equity measure in (26).

The risky asset in (1) shall be given by the German stock index DAX. We use European options written on the DAX, i.e., ODAX quotes, published by the EUREX on 16 January 2013 for the calibration of $S$ and hence, $Y$. Because of the limited number of data we assume that all options with the same maturity $T_{i}, 1 \leq i \leq n$, are characterized by the same volatility $\sigma_{S}\left(T_{i}\right)$ and that the deterministic function $\sigma_{S}(t), t \geq 0$, is piecewise constant, i.e., $\sigma_{S}(t)=\sigma_{S}^{i} \forall t \in\left(T_{i-1}, T_{i}\right]$ for all $1 \leq i \leq n$ with $T_{0}:=0$. For $t=0$ the preceding option pricing formula can be transformed to:

$$
\text { Option }\left(0, T_{i}, S, K, \psi\right)=\psi S(0) \Phi\left(\psi d_{1}\left(T_{i}\right)\right)-\psi K P\left(0, T_{i}\right) \Phi\left(\psi d_{2}\left(T_{i}\right)\right),
$$

where $\forall i \in\{1, \ldots, n\}$ we get for $d_{1}\left(T_{i}\right)$ and $d_{2}\left(T_{i}\right)$ :

$$
\begin{aligned}
& d_{1}\left(T_{i}\right)=\frac{\ln \left(\frac{S(0)}{K \cdot P\left(0, T_{i}\right)}\right)+\frac{1}{2} V\left(Y\left(T_{i}\right)\right)}{\sqrt{V\left(Y\left(T_{i}\right)\right)}} \\
& d_{2}\left(T_{i}\right)=d_{1}\left(T_{i}\right)-\sqrt{V\left(Y\left(T_{i}\right)\right)} .
\end{aligned}
$$

For all $i \in\{1, \ldots, n\}$ it holds for $V\left(Y\left(T_{i}\right)\right)$ that:

$$
\begin{aligned}
V\left(Y\left(T_{i}\right)\right) & =\frac{\sigma_{r}^{2}}{a_{r}^{3}}\left[a_{r} T_{i}-\frac{3}{2}+2 \exp \left(-a_{r} T_{i}\right)-\frac{1}{2} \exp \left(-2 a_{r} T_{i}\right)\right]+\sum_{k=1}^{i}\left(\sigma_{S}^{k}\right)^{2}\left(T_{k}-T_{k-1}\right) \\
& +2 \rho_{S r} \frac{\sigma_{r}}{a_{r}} \sum_{k=1}^{i} \sigma_{S}^{k}\left[\left(T_{k}-T_{k-1}\right)+\frac{1}{a_{r}}\left(\exp \left(a_{r}\left(T_{k-1}-T_{i}\right)\right)-\exp \left(a_{r}\left(T_{k}-T_{i}\right)\right)\right)\right] .
\end{aligned}
$$

For reasons of simplicity, we use the historical correlation between the (simple) daily DAX returns and the 6 month EURIBOR rates as an approximation for $\rho_{S r}$. However, more sophisticated models for $\rho_{S r}$ could be part of future research. Tables 2 and 3 summarize the derived calibration results of the financial market model. 
Table 2. Parameters of the Financial Market Model.

\begin{tabular}{ccc}
\hline$a_{r}$ & $\sigma_{r}$ & $\rho_{S r}$ \\
\hline 0.0799 & 0.0079 & -0.0403 \\
\hline
\end{tabular}

Table 3. Estimated Standard Deviations for DAX Index.

\begin{tabular}{ccccccc}
\hline $\boldsymbol{T}_{\boldsymbol{i}}$ & $\mathbf{0 1 / 1 8 / 2 0 1 3}$ & $\mathbf{0 2 / 1 5 / 2 0 1 3}$ & $\mathbf{0 3 / 1 5 / 2 0 1 3}$ & $\mathbf{0 6 / 2 1 / 2 0 1 3}$ & $\mathbf{0 9 / 2 0 / 2 0 1 3}$ & $\mathbf{1 2 / 2 0 / 2 0 1 3}$ \\
\hline$\sigma_{S}\left(T_{i}\right)$ & 0.1368 & 0.1232 & 0.1557 & 0.1712 & 0.1898 & 0.1993 \\
\hline $\boldsymbol{T}_{\boldsymbol{i}}$ & $\mathbf{0 6 / 2 0 / 2 0 1 4}$ & $\mathbf{1 2 / 1 9 / 2 0 1 4}$ & $\mathbf{0 6 / 1 9 / 2 0 1 5}$ & $\mathbf{1 2 / 1 8 / 2 0 1 5}$ & $\mathbf{1 2 / 1 6 / 2 0 1 6}$ & $\mathbf{1 2 / 1 5 / 2 0 1 7}$ \\
\hline$\sigma_{S}\left(T_{i}\right)$ & 0.2179 & 0.2146 & 0.2367 & 0.2624 & 0.2432 & 0.2237 \\
\hline
\end{tabular}

\subsection{Mortality Model}

In Sections 2.2 and 3.2 we do not discuss the real-world and risk-neutral survival probabilities in detail, as the respective $\mathbb{P}$ - and $\mathbb{Q}$-survival probabilities are heavily affected by the model for the mortality risk premium. This is the reason why both should be part of this calibration section.

Assume $0 \leq t \leq T$. Then, [34] provide the $\mathbb{P}$-survival probability based on (2):

$$
\begin{aligned}
\mathbb{P}\left(\tau^{m}(x)>T \mid \mathcal{G}_{t}\right) & =\mathbb{E}_{\mathbb{P}}\left[\exp \left(-\int_{t}^{T} \lambda_{s}^{m}(x+s) \mathrm{d} s\right) \mid \mathcal{G}_{t}\right] \\
& =\exp \left(A_{x}^{\mathbb{P}}(t, T)+B_{x}^{\mathbb{P}}(t, T) \lambda_{t}^{m}(x+t)\right),
\end{aligned}
$$

with

$$
\begin{aligned}
A_{x}^{\mathbb{P}}(t, T) & =\frac{c_{1} \exp \left(c_{2} T\right)}{c_{3}\left(c_{2}+c_{3}\right)}\left[1-\exp \left(\left(c_{2}+c_{3}\right)(t-T)\right)\right]+\frac{1}{4}\left(\frac{c_{4}}{c_{3}}\right)^{2} \frac{\exp \left(2 c_{5} T\right)}{c_{5}}\left[1-\exp \left(2 c_{5}(t-T)\right)\right] \\
& -\left(\frac{c_{4}}{c_{3}}\right)^{2} \frac{\exp \left(2 c_{5} T\right)}{2 c_{5}+c_{3}}\left[1-\exp \left(\left(2 c_{5}+c_{3}\right)(t-T)\right)\right]-\frac{c_{1} \exp \left(c_{2} T\right)}{c_{2} c_{3}}\left[1-\exp \left(c_{2}(t-T)\right)\right] \\
& +\frac{1}{4}\left(\frac{c_{4}}{c_{3}}\right)^{2} \frac{\exp \left(2 c_{5} T\right)}{c_{3}+c_{5}}\left[1-\exp \left(2\left(c_{3}+c_{5}\right)(t-T)\right)\right] \\
B_{x}^{\mathbb{P}}(t, T) & =\frac{1}{c_{3}}\left[\exp \left(c_{3}(t-T)\right)-1\right]
\end{aligned}
$$

Using [34] the $\mathbb{Q}$-survival probability based on (3) is given by:

$$
\begin{aligned}
\mathbb{Q}\left(\tau^{m}(x)>T \mid \mathcal{G}_{t}\right) & =\mathbb{E}_{\mathbb{Q}}\left[\exp \left(-\int_{t}^{T} \lambda_{s}^{m}(x+s) \mathrm{d} s\right) \mid \mathcal{G}_{t}\right] \\
& =\exp \left(A_{x}^{\mathbb{Q}}(t, T)+B_{x}^{\mathbb{Q}}(t, T) \lambda_{t}^{m}(x+t)\right),
\end{aligned}
$$

with

$$
\begin{aligned}
A_{x}^{\mathbb{Q}}(t, T) & =A_{x}^{\mathbb{P}}(t, T)+\frac{c_{4}}{c_{3} c_{5}} \gamma_{m}\left(\exp \left(c_{5} T\right)-\exp \left(c_{5} t\right)\right)-\frac{c_{4}}{c_{3}\left(c_{3}+c_{5}\right)} \gamma_{m} \exp \left(c_{5} T\right) \\
& +\frac{c_{4}}{c_{3}\left(c_{3}+c_{5}\right)} \exp \left(-c_{3} T\right) \gamma_{m} \exp \left(\left(c_{5}+c_{3}\right) t\right), \\
B_{x}^{\mathbb{Q}}(t, T) & =\frac{1}{c_{3}}\left[\exp \left(c_{3}(t-T)\right)-1\right] .
\end{aligned}
$$


For a fixed age $x$, and $0 \leq t \leq T$, let $\mathrm{M}_{x}(t, T)$ be defined by:

$$
\begin{aligned}
\mathrm{M}_{x}(t, T) & =\ln \left(\frac{\mathbb{Q}\left(\tau^{m}(x)>T \mid \mathcal{G}_{t}\right)}{\mathbb{P}\left(\tau^{m}(x)>T \mid \mathcal{G}_{t}\right)}\right) \\
& =\frac{c_{4}}{c_{3} c_{5}} \gamma_{m}\left(\exp \left(c_{5} T\right)-\exp \left(c_{5} t\right)\right)-\frac{c_{4}}{c_{3}\left(c_{3}+c_{5}\right)} \gamma_{m} \exp \left(c_{5} T\right) \\
& +\frac{c_{4}}{c_{3}\left(c_{3}+c_{5}\right)} \exp \left(-c_{3} T\right) \gamma_{m} \exp \left(\left(c_{5}+c_{3}\right) t\right)
\end{aligned}
$$

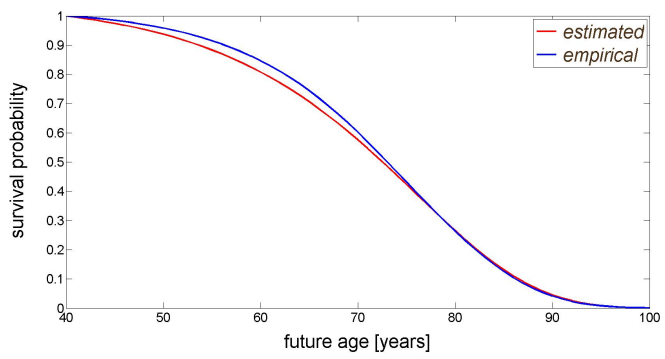

(a)

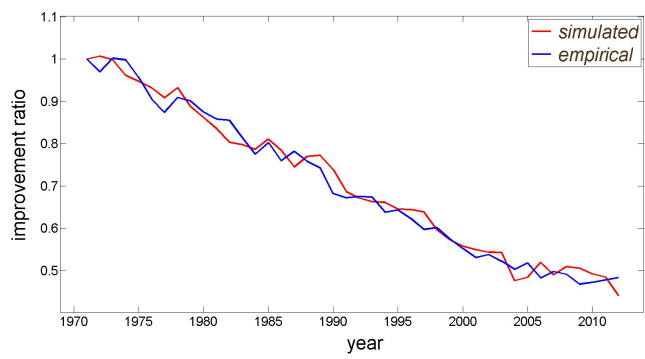

(b)

Figure 4. Gompertz Model (a) and Improvement Ratio (b) for Men Aged 40 Years in 1971.

Figure 4 displays the calibration results for the real-world measure $\mathbb{P}$ which are derived from U.K. mortality data. On the left we show the empirical survival probabilities (blue) and their analogs based on the estimated, static Gompertz model (red). The decreasing blue line on the right indicates that mortality for the given group is generally improving. The mortality improvement ratio $\xi$ is modeled as an Ornstein-Uhlenbeck process, this is why the red line on the right-hand side illustrates an arbitrary trajectory. As there is no liquid market for actively traded annuities (especially not for variable ones), the estimation of the mortality risk premium $\gamma_{m}$ has to rely on primary market prices. For this purpose, the annual averages of level annuity rates (equipped with a five-year guarantee, offered to men aged 65 years in the U.K.) provided by [37] serve as an approximation and are displayed in the left plot of Figure 6. To enter a level annuity contract a policyholder has to pay a lump sum at the beginning. Thereafter, he is entitled to a constant (annual) repayment for his remaining lifetime. Consequently, there are no adjustments caused by inflation, an overperforming fund, etc. to be addressed. If $\gamma_{m}$ is supposed to be constant depending on the year a contract was issued and the age of an insured, we get for a contract issued in $u=$ calendar year -1971 :

$$
\frac{I_{u}}{L_{u}}=\sum_{k=1}^{5} P(u, u+k)+\sum_{k=6}^{T^{*}-x} \mathbb{Q}\left(\tau_{u}^{m}(x)>k \mid \mathcal{G}_{u}\right) P(u, u+k) .
$$

The index $u$ in $\tau_{u}^{m}(x)$ denotes the year the contract was issued. $I_{u}$ and $L_{u}$ stand for its initial premium and the agreed annual repayment of the level annuity. Using (30), the mortality tables of Figure 1 (upper limit in age is 101 years) and the fact that a person aged $x$ in $u$ was $x-u$ in 1971 it follows that:

$$
\frac{I_{u}}{L_{u}}=\sum_{k=1}^{5} P(u, u+k)+\sum_{k=6}^{36}\left[\mathbb{P}\left(\tau_{u}^{m}(x)>k \mid \mathcal{G}_{u}\right) \exp \left(\mathrm{M}_{x-u}(u, u+k)\right) P(u, u+k)\right] .
$$

The annuities in [37] have been offered in the U.K. implying that the estimated financial market model based on EURIBOR rates and DAX cannot be used for pricing the bonds $P(u, u+k)$. We aim to show how the chosen mortality risk premium may be estimated. In doing so, we do not claim that this solution is the one and only way. Depending on the intention and needs of the user alternative 
approaches might be more appropriate. We replace $P(u, u+k)$ through the corresponding discount factors derived from the nominal rates of U.K. government bonds ${ }^{6}$ published by the Bank of England and illustrated in Figure 5. Besides the nominal rates of U.K. government bonds, the discount factors of an insurance company possibly take into account corporate bond yields, and equity and other returns, which significantly affects the estimated mortality risk premium $\gamma_{m}$. Across the full range of insurance companies and annuity products, the internal discount factors may vary considerably. As [37] provide averaged annuity rates which are not restricted to a single provider, we cannot construct discount factors covering the entire annuity business. Furthermore, as soon as the discount factors are based on some defaultable products such as corporate bonds the inherent default risk has to be incorporated and separated from the mortality risk. If a risk-free rate is used for discounting, there are no default risks requiring particular attention. Concerning the annuity rates in [37], the nominal rates of U.K. government bonds might be a good approximation for the risk-free rate as their default risk is quite low and each insurance company is able to invest in them. Using linear interpolation whenever inner maturities are missing and constant extrapolation for maturities greater than 25 years results in the term structures displayed in Figure 5. The estimated parameters of the mortality model are summarized in Table 4.

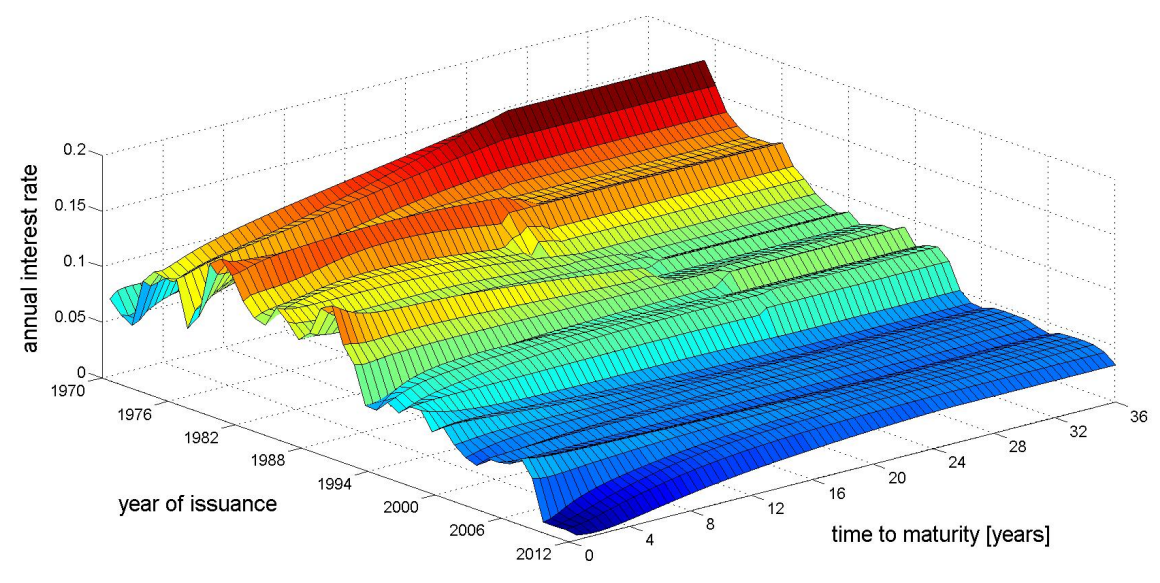

Figure 5. U.K. Nominal Rates (1970-2012).

Table 4. Mortality Parameters

\begin{tabular}{ccccccc}
\hline Parameter & $\boldsymbol{b}$ & $\boldsymbol{z}$ & $\boldsymbol{\kappa}$ & $\gamma$ & $\sigma_{\tilde{\zeta}}$ & $\gamma_{m}$ \\
\hline Estimated Value & 12.1104 & 76.1390 & 0.4806 & 0.0195 & 0.0254 & 10.6482 \\
\hline
\end{tabular}

On the one hand, Figure 4 confirms that the combination of the static Gompertz model and the mortality improvement ratio, i.e., the mortality model under $\mathbb{P}$, is able to properly map the real-world mortality tables. On the other hand, the plot on the left of Figure 6 indicates that the constant mortality risk premium $\gamma_{m}$ and the nominal rates of the U.K. government bonds are suitable for the replication of the annuity rates of [37], as there are only minor deviations between the original annuity rates and their theoretical counterparts based on the estimated parameters of Table 4 . All in all, the mortality model provides convincing results regarding the real-world measure $\mathbb{P}$ and the risk-neutral pricing measure $\mathbb{Q}$. However, the interpretation of $\gamma_{m}=10.6482$ is still missing. Using the plot on the right in Figure 6 we can conclude: First, the blue and red curves are shaped similarly such that the constant

6 The data can be downloaded from http://www.bankofengland.co.uk/statistics/Pages/yieldcurve/archive.aspx. 
mortality risk premium $\gamma_{m}$ does not change the structure of the survival probabilites; Second, for any fixed age the risk-neutral survival probability exceeds its real-world analog. From the perspective of an annuity provider this makes sense. The $\mathbb{P}$-survival probabilities arise from the real mortality tables, whereas the $\mathbb{Q}$-survival probabilities are used for pricing annuities. If the difference between the $\mathbb{Q}$ - and $\mathbb{P}$-survival probabilities is positive, an annuity provider implicitly assumes in his annuity prices that the insureds live on average longer than the mortality tables reflect. So, in addition to the mortality risk derived from real mortality tables, a premium in the form of the spread between the $\mathbb{Q}$ - and $\mathbb{P}$-survival probabilities is charged by the insurance company for taking the insureds' longevity risk. Within our modeling framework $\gamma_{m}$ covers the magnitude and direction of this longevity risk premium. The larger the absolute value of $\gamma_{m}$ the larger the absolute value of the spread between both survival probabilities. Whenever $\gamma_{m}$ is negative, the $\mathbb{Q}$-survival probabilities are smaller than their $\mathbb{P}$-analogues. For instance, in case of term life assurances, insurance companies are facing the opposite risk, i.e., that the insureds die earlier than expected.

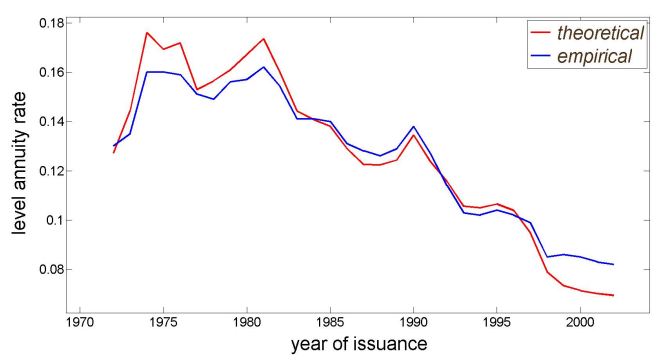

(a)

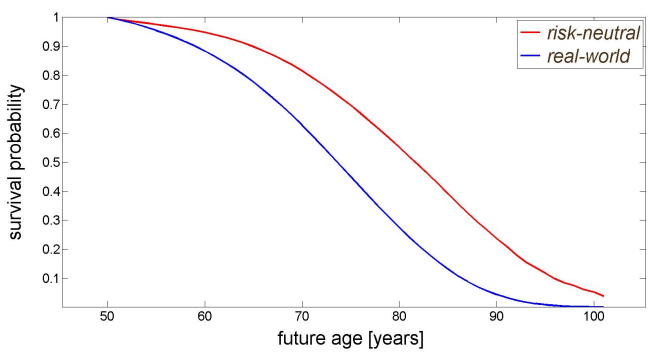

(b)

Figure 6. Level Annuities of [37] (a) and Survival Probabilities (b).

\subsection{Surrender Model}

For $1 \leq i \leq K$, let $q_{i}$ denote the probability that the early surrender option is exercised before $t_{i}$. To be precise, let $q_{1}, \ldots, q_{K}$ represent the (hypothetical) surrender probabilities we want to use for product pricing. Then, (27) provides for all $1 \leq i \leq K$ :

$$
1-q_{i} \approx \exp \left(-C \cdot t_{i}\right) \cdot S_{11}^{\mathbb{Q}^{S\left(t_{i}\right)}}\left(D, \alpha, \beta, \tilde{\mathbf{t}}_{\mathbf{i}}\right) .
$$

Using (19) and (20), we obtain:

$$
\begin{aligned}
S_{11}^{\mathbb{Q}^{S\left(t_{i}\right)}}\left(D, \alpha, \beta, \tilde{\mathbf{t}}_{\mathbf{i}}\right)=1 & +\sum_{j=1}^{i} \exp \left(\bar{h}_{1}\left(\beta, D, t_{j}\right)\right) \Phi\left(\bar{h}_{2}\left(0, \beta, D, t_{j}\right)\right)-\sum_{j=1}^{i} \exp \left(\bar{h}_{1}\left(\beta, D, t_{j}\right)\right) \Phi\left(\bar{h}_{2}\left(\alpha, \beta, D, t_{j}\right)\right) \\
& +\sum_{j=1}^{i} \exp \left(-\beta \alpha \Delta t_{j}\right) \Phi\left(\bar{h}_{2}\left(\alpha, 0, D, t_{j}\right)\right)-\sum_{j=1}^{i} \Phi\left(\bar{h}_{2}\left(0,0, D, t_{j}\right)\right),
\end{aligned}
$$

where it holds for all $1 \leq j \leq i$ :

$$
\begin{aligned}
\bar{h}_{1}\left(\beta, D, t_{j}\right): & =-\beta \Delta t_{j} \mathbb{E}_{\mathbb{Q}^{S\left(t_{i}\right)}}\left[D\left(t_{j}\right)\right]+\frac{1}{2} \beta^{2} \Delta t_{j}^{2} \operatorname{Var}_{\mathbb{Q}^{S\left(t_{i}\right)}}\left[D\left(t_{j}\right)\right], \\
\bar{h}_{2}\left(\alpha, \beta, D, t_{j}\right): & =\frac{\mathbb{E}_{\mathbb{Q}^{S\left(t_{i}\right)}\left[D\left(t_{j}\right)\right]-\alpha}}{\sqrt{\operatorname{Var}_{\mathbb{Q}^{S\left(t_{i}\right)}}\left[D\left(t_{j}\right)\right]}}-\beta \Delta t_{j} \sqrt{\operatorname{Var}_{\mathbb{Q}^{S\left(t_{i}\right)}}\left[D\left(t_{j}\right)\right]} .
\end{aligned}
$$

The moments $\mathbb{E}_{\mathbb{Q}^{S\left(t_{i}\right)}}\left[D\left(t_{j}\right)\right]$ and $\operatorname{Var}_{\mathbb{Q}^{S\left(t_{i}\right)}}\left[D\left(t_{j}\right)\right]$ are given in Appendix A.1. After the calibration results of the financial market have been determined, an ordinary least-squares regression yields the estimation of the unknown surrender parameters $\alpha, \beta$ and $C$.

Due to a lack of data, the hypothetical parameters in Table 5 are considered. Case 1 neglects early surrender such that we are able to analyze the value of the surrender option and to test the quality of 
the approximation without any disturbances resulting from the surrender add-on. The parameters of Case 2 and Case 3 were chosen to get first-year surrender probabilities within a range of $1 \%-5 \%$ and $5 \%-10 \%$, respectively ${ }^{7}$. The first-year surrender intensities based on Case 2 and Case 3 are illustrated in Figure 7. For the remaining drivers of the decision criterion $D$, i.e., $\delta, f$ and $T$, see Table 6.

Table 5. Parameters of Surrender Model.

\begin{tabular}{cccc}
\hline & Case 1 & Case 2 & Case 3 \\
\hline$\alpha$ & 0.00 & 1.00 & 0.25 \\
$\beta$ & 0.00 & 0.04 & 0.20 \\
$C$ & 0.00 & 0.01 & 0.05 \\
\hline
\end{tabular}

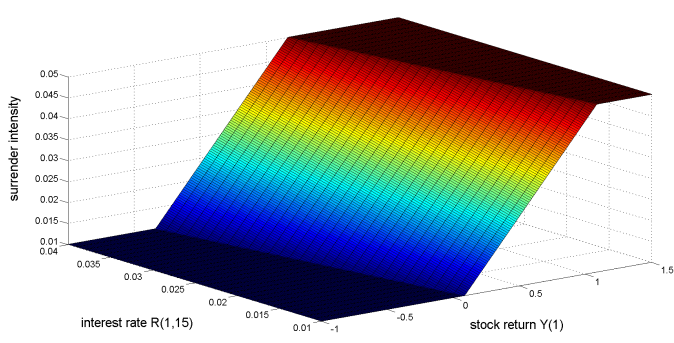

(a)

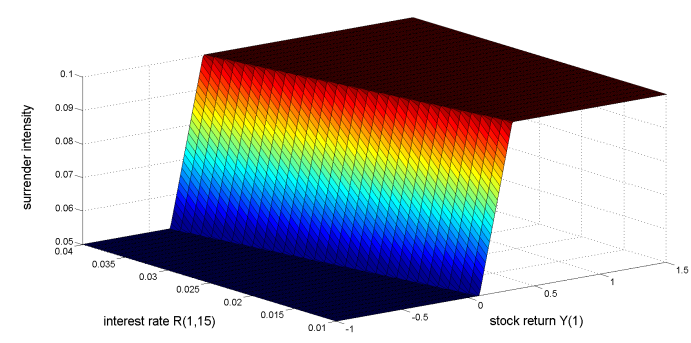

(b)

Figure 7. First-Year Surrender Probability 1\%-4.88\% (a) and 4.88\%-9.52\% (b).

Table 6. Specification of Variable Annuity (VA) Contract.

\begin{tabular}{cc}
\hline Insured & man, aged 50 years \\
\hline Premium & 100 \\
\hline Maturity & 15 years \\
\hline$\delta$ & $1.00 \%$ \\
\hline Termination dates & $1, \ldots, 14$ (once per year) \\
\hline Repayment Dates & $1, \ldots, 15$ (once per year) \\
\hline Surrender Fees & $\begin{array}{c}7 \%, \ldots, 1 \% \text { (years } 1-7, \text { linear) } \\
0 \% \text { (years } 8-14, \text { no fee) }\end{array}$ \\
\hline
\end{tabular}

\section{Numerical Example}

To check the quality of the presented pricing framework, in particular, to detect its errors and limitations we price the VAs in (9) twice, that is, using our closed-form approximation and MC. The simulated prices will serve as a benchmark for the approximated ones; we generate 500,000 trajectories of the processes $Y$ and $r$ on a monthly basis. For the model parameters the calibration results in Tables 2-5 are taken. The contract specification of the considered VA is defined in Table 6.

The authors of [6] derive an analytic solution for GMABs in the absence of early surrender. Since the work in (28) provides a closed-form solution for GMDBs and the value of the SB is zero, for Case 1 of Table 5 an analytic solution exists. The positive $\gamma_{m}$ in Table 4 implies that the $\mathbb{Q}$-survival probabilities exceed their $\mathbb{P}$-counterparts (see Figure 6) reflecting the premium charged for longevity risk. In the

7 For the parameters in Table 5 the actual boundaries are $1 \%-4.88 \%$ and $4.88 \%-9.82 \%$. 
case of GMDBs an insurer is facing the reverse risk, i.e., that an insured dies earlier than expected. Here, we keep the $\gamma_{m}$ from Table 4 for the pricing of the GMDB, as we are not able to estimate the risk premium due to missing data. A summary of the priced VAs is displayed in Table 7.

Table 7. VA Prices, Standard Deviations and Differences.

\begin{tabular}{|c|c|c|c|}
\hline \multicolumn{4}{|c|}{ GMAB } \\
\hline & Case 1 & Case 2 & Case 3 \\
\hline Analytic Sol. & 110.6804 & $\mathrm{x}$ & $x$ \\
\hline Approximation & 110.6804 & 79.2336 & 36.2581 \\
\hline Sim. (mean, 500K) & 110.9925 & 81.2484 & 39.0351 \\
\hline Sim. (std, 500K) & 0.1200 & 0.0662 & 0.0303 \\
\hline Delta (abs.) ${ }^{8}$ & -0.3121 & -2.0148 & -2.7771 \\
\hline Delta (rel.) $)^{9}$ & -0.0028 & -0.0248 & -0.0711 \\
\hline \multicolumn{4}{|c|}{ SB } \\
\hline & Case 1 & Case 2 & Case 3 \\
\hline Analytic Sol. & 0 & $x$ & $x$ \\
\hline Approximation & 0 & 26.3468 & 62.9797 \\
\hline Sim. (mean, 500K) & 0 & 27.7328 & 63.2259 \\
\hline Sim. (std, 500K) & 0 & 0.0397 & 0.0519 \\
\hline Delta (abs.) & 0 & -1.3860 & -0.2462 \\
\hline Delta (rel.) & $x$ & -0.0500 & -0.0039 \\
\hline \multicolumn{4}{|c|}{ GMDB } \\
\hline & Case 1 & Case 2 & Case 3 \\
\hline Analytic Sol. & 12.2560 & $x$ & $x$ \\
\hline Approximation & 12.2560 & 10.3374 & 6.9443 \\
\hline Sim. (mean, 500K) & 12.2589 & 10.4157 & 7.0785 \\
\hline Sim. (std, 500K) & 0.0078 & 0.0049 & 0.0027 \\
\hline Delta (abs.) & -0.0029 & -0.0783 & -0.1343 \\
\hline Delta (rel.) & -0.0002 & -0.0075 & -0.0190 \\
\hline \multicolumn{4}{|c|}{$\mathrm{VA}=\mathrm{GMAB}+\mathrm{SB}+\mathrm{GMDB}$} \\
\hline & Case 1 & Case 2 & Case 3 \\
\hline Analytic Sol. & 122.9363 & $\mathrm{x}$ & $x$ \\
\hline Approximation & 122.9363 & 115.9177 & 106.1820 \\
\hline Sim. (mean, 500K) & 123.2514 & 119.3968 & 109.3395 \\
\hline Sim. (std, 500K) & 0.1270 & 0.1007 & 0.0731 \\
\hline Delta (abs.) & -0.3150 & -3.4791 & -3.1575 \\
\hline Delta (rel.) & -0.0026 & -0.0291 & -0.0289 \\
\hline
\end{tabular}

Table 7 shows that high first-year surrender probabilities (Case 1: 0\%; Case 2: 1\%-5\%; Case 3: $5 \%-10 \%$ ) reduce the GMAB prices. An increased surrender probability decreases the likelihood that a policyholder is entitled to the GMAB payoff, justifying the price decline. A high termination probability makes claims from the SB more likely (prices increase). A high likelihood of surrender enhances the chance that a policyholder surrenders his contract before he dies so that the decreased GMDB prices are reasonable. If early surrender occurs, a policyholder's right of refund is restricted to the fund reduced by the prevailing surrender fee. The absence of any guarantee diminishes the financial distress 
of the insurance company compared to the GMAB and GMDB. The theoretical assumption that the increase in the SB prices is smaller than the aggregated decrease in the GMAB and GMDB prices is supported by the decreasing VA prices.

In Case 1, the analytic solution and the closed-form approximation provide the same prices indicating that our approach works properly, if early surrender is neglected. MC prices of GMABs and GMDBs are slightly different, but these small deviations are acceptable. In the sequel, the MC prices will serve as a benchmark for their approximated counterparts. Table 7 shows for GMABs, SBs and GMDBs an increase in the absolute error when their values decline. That is to say, the less the worth of a component is the higher its pricing error becomes. This is important, since the most valuable constituents are properly priced. Due to (28) a GMDB may be regarded as a portfolio of GMABs. Therefore, it is no coincidence that for all cases the pricing errors of GMABs and GMDBs have the same sign. Note that for the overall VA the absolute value of Delta (rel.) remains smaller than $3.00 \%$ for all considered cases.

For a better understanding of the assumed surrender behavior Figure 8 illustrates for all termination dates the empirical means of the simulated surrender intensities. Recalling Table 5 we can see that both time series meet the initial conditions. The surrender intensity of Case 2 is increasing while the one of Case 3 does the opposite. In both cases the same decision criterion is applied, but different parametrizations of the surrender intensity result in different admissible upper and lower bounds. In total, they cause distinct s-curves such that the simulated decision criterion reaches/exceeds their upper and lower limits in different scenarios.

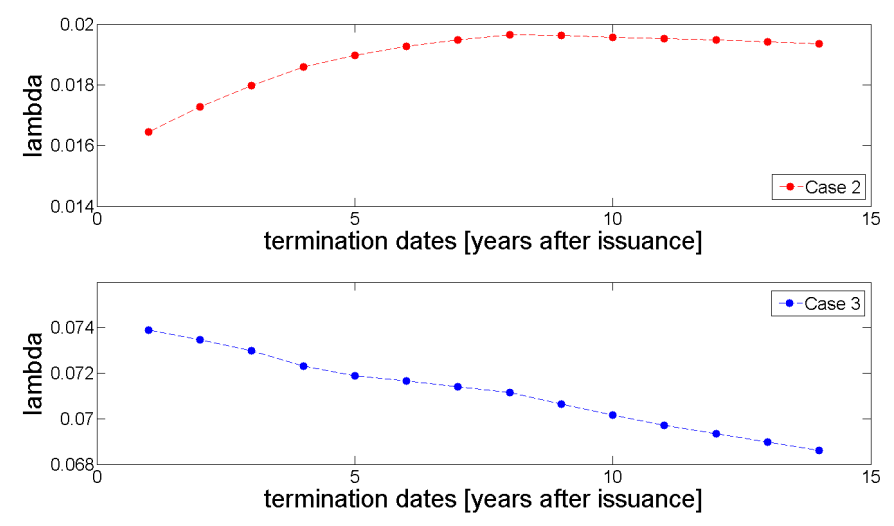

Figure 8. Means of Simulated Surrender Intensities

Finally, we briefly repeat the main advantage of the presented pricing scheme. Simulation techniques often require a sufficient number of scenarios together with an appropriate grid size (e.g., monthly steps). This may call for a lot of computational power and can be quite time consuming. By contrast, the derived closed-form approximation requires simple calculations. Each run takes a fraction of the simulation time, and thus, causes significant savings in computation time.

\section{Emergency Fund Extension}

The emergency fund hypothesis assumes that personal financial distress or other factors (e.g., unemployment or illness) force policyholders to prematurely surrender their contracts. In the sequel, let a major drop in stock market prices be one of these factors resulting in financial distress for some policyholders (in addition to the ones already covered by the lower limit $C$ ). To be precise, let the time-dependent function $l:[0, T] \rightarrow[-\infty, 0]$ denote the lower limit at time $t$ triggering additional surrender arising from a badly performing fund. Then, for any point in time $t \in[0, T]$ with $Y(t)<l(t)$ we have an increased surrender intensity. In general, a steep decline in stock market prices should exert pressure on more policyholders than a small one does. Thus, we assume for any fixed $t \in[0, T]$ 
the more $Y(t)$ decreases on the interval $Y(t) \in[-\infty, l(t)]$, i.e., the bigger the loss on the fund account, the more the surrender intensity increases up to an upper limit. For an illustration of the new surrender intensity $\tilde{\lambda}^{s}(t)$ based on different parameters see Figure 9. If we assume a piecewise linear relation between $Y(t)$ and $\tilde{\lambda}^{s}(t)$ in the form of two s-curves (one covering the policyholders' surrender behavior described in Section 2.3 and one covering the emergency fund hypothesis), for any fixed point in time $t \in[0, T]$ we get:

$$
\tilde{\lambda}^{s}(t):=\beta \max [\min [D(t), \alpha], 0]-\tilde{\beta} \max [\min [\tilde{D}(t), \tilde{\alpha}], 0]+\tilde{C},
$$

with $\alpha, \tilde{\alpha}, \beta, \tilde{\beta}, \tilde{C} \in \mathbb{R}_{0}^{+}$and $\tilde{D}(t):=Y(t)-l(t)$.

Because of its relevance for applications in practice we show how the surrender model definded by Case 2 in Table 5 can be properly extended to the emergency fund hypothesis. On the interval $[0, \infty]$ the behavior of new intensity $\tilde{\lambda}^{s}$ shall coincide with the behavior of the old intensity $\lambda^{s}$. Therefore, we keep the parameters $\alpha$ and $\beta$ of Table 5 . The constant $\tilde{C}$ has to be adjusted to end up with the original lower limit $C$. For this purpose, $\tilde{C}$ has to satisfy:

$$
\tilde{C}-\tilde{\alpha} \tilde{\beta}=C .
$$

Since we are not able to calibrate this extended surrender model due to the lack of appropriate surrender data, we use the hypothetical values in Table 8 . The corresponding surrender intensities are displayed in Figure 9. Using the contract specification in Table 6 we obtain the probabilities in Figure 10. These surfaces present the probability that the early surrender option is not exercised within the first year depending on the accumulated stock return $Y(1)$ and long-term interest rate $R(1,15)$.

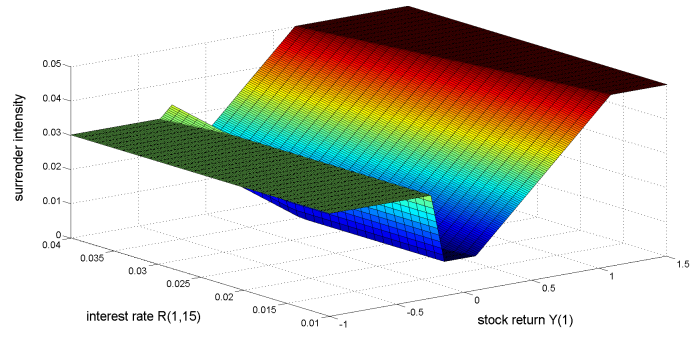

(a)

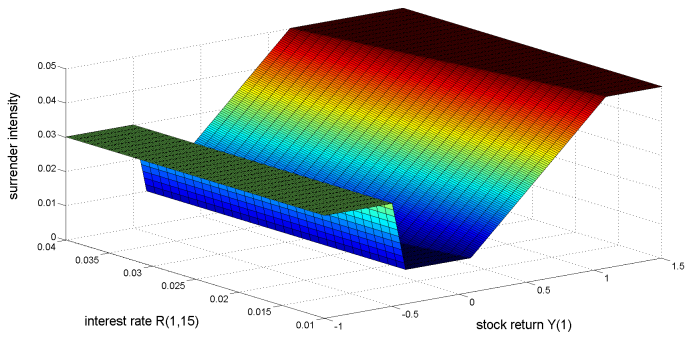

(b)

Figure 9. Surrender Intensities of Cases 4 (a) and 5 (b).

The only difference between Cases 4 and 5 is given by the lower limit $l(t) \equiv l$ which has to be explained, in particular, regarding the assumed surrender behavior of the policyholders. In Case 4 we have that if the value of the fund drops to $86.07 \%=\exp (-0.15)$ of the initial investment, the emergency add-on is activated. As soon as the fund further declines to $77.88 \%=\exp (-0.25)$ the one-year surrender probability increase from $1 \%$ to $2.96 \%$ where it is capped. Similarly, we have in Case 5 that if the value of the fund drops from $67.03 \%=\exp (-0.40)$ to $60.65 \%=\exp (-0.50)$, the one-year surrender probability increases from $1 \%$ to $2.96 \%$ where it is again capped. Hence, in Case 4 additional surrender due to the emergency fund hypothesis is triggered earlier, indicating that the policyholders characterized by Case 4 suffer more from a downturn on the stock market than the ones described by Case 5. In both scenarios a negative fund performance causes an increase in the one-year surrender probability of almost $2 \%$. 


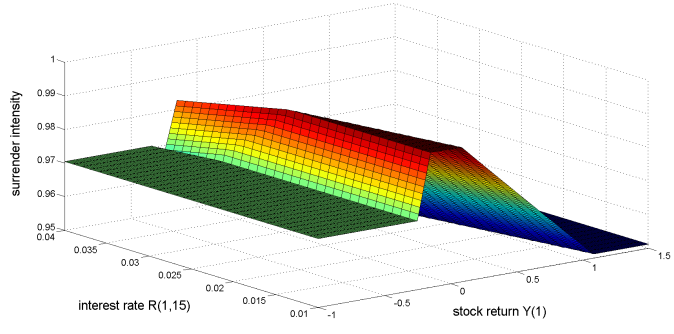

(a)

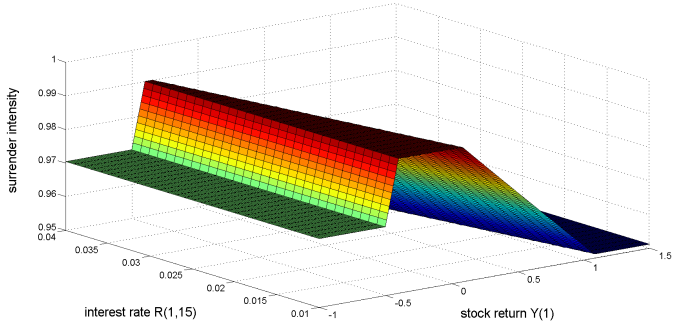

(b)

Figure 10. Survival Probabilities of Cases 4 (a) and 5 (b).

Table 8. Parameters of the Extended Surrender Model.

\begin{tabular}{ccc}
\hline & Case 4 & Case 5 \\
\hline$\alpha$ & 1.00 & 1.00 \\
$\beta$ & 0.04 & 0.04 \\
$\tilde{\alpha}$ & 0.10 & 0.10 \\
$\tilde{\beta}$ & 0.20 & 0.20 \\
$\tilde{C}$ & 0.03 & 0.03 \\
$l$ & -0.25 & -0.50 \\
\hline
\end{tabular}

Using (10) we can price GMABs based on $\tilde{\lambda}^{s}$. As $\lambda^{s}$ enters $E_{1}\left[\lambda^{s}\right]$ and $E_{2}\left[\lambda^{s}\right]$ only, we just have to derive equivalent solutions for $E_{1}\left[\tilde{\lambda}^{s}\right]$ and $E_{2}\left[\tilde{\lambda}^{s}\right]$. To do this, we apply the same methods as before and get:

$$
\begin{aligned}
& E_{1}\left[\tilde{\lambda}^{s}\right] \approx \exp \left(-\tilde{C} \cdot t_{K}\right) \cdot\left(\tilde{S}_{11}^{\mathbb{Q}^{T}}(D, \tilde{D}, \alpha, \tilde{\alpha}, \beta, \tilde{\beta}, \mathbf{t})+\tilde{S}_{12}^{\mathbb{Q}^{T}}(D, \tilde{D}, \alpha, \tilde{\alpha}, \beta, \tilde{\beta}, \mathbf{t})\right), \\
& E_{2}\left[\tilde{\lambda}^{s}\right] \approx \exp \left(-\tilde{C} \cdot t_{K}\right) \cdot \tilde{S}_{2}^{\mathbb{Q}^{T}}(D, \tilde{D}, \tilde{Y}, \alpha, \tilde{\alpha}, \beta, \tilde{\beta}, \mathbf{t}),
\end{aligned}
$$

with

$$
\begin{aligned}
\tilde{S}_{11}^{\mathbb{Q}^{T}}(D, \tilde{D}, \alpha, \tilde{\alpha}, \beta, \tilde{\beta}, \mathbf{t}) & =S_{11}^{\mathbb{Q}^{T}}(D, \alpha, \beta, \mathbf{t})-1+S_{11}^{\mathbb{Q}^{T}}(\tilde{D}, \tilde{\alpha},-\tilde{\beta}, \mathbf{t}), \\
\tilde{S}_{12}^{\mathbb{Q}^{T}}(D, \tilde{D}, \alpha, \tilde{\alpha}, \beta, \tilde{\beta}, \mathbf{t}) & =S_{12}^{\mathbb{Q}^{T}}(D, \alpha, \beta, \mathbf{t})+S_{12}^{\mathbb{Q}^{T}}(\tilde{D}, \tilde{\alpha},-\tilde{\beta}, \mathbf{t}), \\
\tilde{S}_{2}^{\mathbb{Q}^{T}}(D, \tilde{D}, \tilde{Y}, \alpha, \tilde{\alpha}, \beta, \tilde{\beta}, \mathbf{t}) & =S_{2}^{\mathbb{Q}^{T}}(D, \tilde{Y}, \alpha, \beta, \mathbf{t})-\mathbb{E}_{\mathbb{Q}^{T}}\left[\exp (\tilde{Y}(T)) \mathbb{1}_{\{\tilde{Y}(T) \geq 0\}]}\right] \\
& +S_{2}^{\mathbb{Q}^{T}}(\tilde{D}, \tilde{Y}, \tilde{\alpha},-\tilde{\beta}, \mathbf{t})+\mathbb{Q}^{T}(\tilde{Y}(T) \geq 0) .
\end{aligned}
$$

The new pricing formula for GMABs and (28) enable the pricing of GMDBs based on $\tilde{\lambda}^{s}$ without any further calculations. Eventually, the SB pricing in (25) requires the probability that no surrender takes place before a certain point in time. Similar to (27), it follows for $\tilde{\lambda}^{s}$ :

$$
\mathbb{Q}^{S\left(t_{i}\right)}\left(\tilde{\tau}^{S}>t_{i}\right) \approx \exp \left(-\tilde{C} \cdot t_{i}\right) \cdot \tilde{S}_{11}^{\mathbb{Q}^{S\left(t_{i}\right)}}(D, \tilde{D}, \alpha, \tilde{\alpha}, \beta, \tilde{\beta}, \mathbf{t})
$$

where $\tilde{\tau}^{s}$ is the counterpart of $\tau^{s}$ for $\tilde{\lambda}^{s}$.

Table 9 states the simulated and approximated GMAB, SB, GMDB and VA prices for the original surrender intensity $\lambda^{s}$ (Case 2) and its extended version $\tilde{\lambda}^{s}$ (Cases 4 and 5). On the one hand, a comparison between Cases 4 and 5 detects how the lower limit $l$ affects the prices. On the other hand, Case 2 serves as a benchmark such that we are able to analyze the impact of the emergency fund component in $\tilde{\lambda}^{s}$ on the prices. 
Table 9. VA Prices, Standard Deviations and Differences.

\begin{tabular}{|c|c|c|c|}
\hline \multicolumn{4}{|c|}{ GMAB } \\
\hline & Case 2 & Case 4 & Case 5 \\
\hline Approximation & 79.2336 & 75.4547 & 78.1821 \\
\hline Sim. (mean, $500 \mathrm{~K})$ & 81.2484 & 74.6267 & 77.2791 \\
\hline Sim. (std, 500 K) & 0.0662 & 0.0680 & 0.0678 \\
\hline Delta (abs.) & -2.0148 & 0.8280 & 0.9031 \\
\hline Delta (rel.) & -0.0248 & 0.0111 & 0.0117 \\
\hline \multicolumn{4}{|c|}{ SB } \\
\hline & Case 2 & Case 4 & Case 5 \\
\hline Approximation & 26.3468 & 28.5614 & 26.8172 \\
\hline Sim. (mean, $500 \mathrm{~K})$ & 27.7328 & 31.5358 & 29.6096 \\
\hline Sim. (std, 500 K) & 0.0397 & 0.0367 & 0.0381 \\
\hline Delta (abs.) & -1.3860 & -2.9744 & -2.7924 \\
\hline Delta (rel.) & -0.0500 & -0.0943 & -0.0943 \\
\hline \multicolumn{4}{|c|}{ GMDB } \\
\hline & Case 2 & Case 4 & Case 5 \\
\hline Approximation & 10.3374 & 9.9925 & 10.2123 \\
\hline Sim. (mean, $500 \mathrm{~K})$ & 10.4157 & 9.9483 & 10.1630 \\
\hline Sim. (std, 500 K) & 0.0049 & 0.0051 & 0.0050 \\
\hline Delta (abs.) & -0.0783 & 0.0442 & 0.0493 \\
\hline Delta (rel.) & -0.0075 & 0.0044 & 0.0048 \\
\hline \multicolumn{4}{|c|}{$\mathrm{VA}=\mathrm{GMAB}+\mathrm{SB}+\mathrm{GMDB}$} \\
\hline & Case 2 & Case 4 & Case 5 \\
\hline Approximation & 115.9177 & 114.0086 & 115.2116 \\
\hline Sim. (mean, $500 \mathrm{~K})$ & 119.3968 & 116.1108 & 117.0517 \\
\hline Sim. (std, 500 K) & 0.1007 & 0.1017 & 0.1021 \\
\hline Delta (abs.) & -3.4791 & -2.1022 & -1.8401 \\
\hline Delta (rel.) & -0.0291 & -0.0181 & -0.0157 \\
\hline
\end{tabular}

The additional s-curve covering the emergency fund hypothesis increases the SB prices and decreases the GMAB and GMDB prices. If the probability of premature contract termination increases, the probability that a policyholder is entitled to the repayment of the GMAB decreases. Similarly, a higher surrender probability increases the chance that a contract will be surrendered before the death of the insured, reducing the payment obligations of the GMDB. By contrast, the policyholder is more likely to receive the payoffs of the SB.

The prices in Table 9 encourage the aforementioned assumption that termination based on the emergency hypothesis is triggered earlier in Case 4 than in Case 5, as the SB price in Case 4 exceeds its analog in Case 5. The opposite holds for the prices of the GMABs and GMDBs. Although the overall VA is worth more or less the same, the fair values of its single constituents have significantly changed. For instance, compare the GMAB and SB prices in Cases 2 and 4.

In spite of the emergency fund hypothesis, the magnitude of the relative pricing errors has not considerably changed. Hence, the presented approximation is able to properly price the VAs in (9) when additional sources for early surrender are taken into account. Note, this extended example shows once again the modularity and the inherent flexibility of the presented pricing framework which could be particularly useful for practitioners. 


\section{Conclusion and Future Research}

In this paper we construct an affine linear framework with stochastic models for the financial market, the insureds' mortality and the policyholders' surrender behavior. The financial market includes a risk-free interest rate and a risky asset which is supposed to be a stock or a fund. Two distinct intensity based approaches map the insureds' mortality and the policyholders' surrender behavior. A static Gompertz model and an Ornstein-Uhlenbeck process covering the increasing life expectancy of humans form the mortality intensity. Some empirical studies detect an irrationality in the surrender behavior of policyholders. For instance, they surrender their contracts, although it is not rational from the perspective of maximizing portfolio value. Furthermore, some empirical studies confirm the interest-rate hypothesis that rising interest rates cause increased contract termination. To take into account both findings we deploy an s-shaped surrender intensity using a deterministic function with stochastic inputs from the financial market.

We focus on variable annuities requiring an upfront payment which is paid by the policyholder and is fully invested in the risky asset. The surrender benefit strongly affects the behavior of the policyholders. To avoid additional incentives for premature surrender a policyholder's right of refund is restricted to the current value of the risky investment reduced by the prevailing surrender fee. If the insured dies before the early surrender option is exercised, in particular, before the maturity of the contract, the payoff is given by the maximum of the current value of the risky investment and a preliminary agreed minimum benefit. The same choice is offered at maturity.

The main result of this paper is the derived closed-form approximation. Although the financial market, the insureds' mortality and the policyholders' surrender behavior are stochastic, we price certain variable annuities using a closed-form approximation. Despite some lengthy expressions, the presented pricing framework can be easily implemented. Compared to simulation techniques it requires less computational power and time, which could be particularly important for applications in practice. Additionally, we show how the stochastic models can be calibrated using actively traded financial products and historic mortality data. Due to missing data the calibration of the surrender model is based on hypothetical values. All in all, the displayed solution represents one possible way of calibrating the models. Depending on the needs of the user and the area of application (risk management, pricing, etc.) alternative calibration methods could be preferred. The usage of actively traded products supports subsequent hedging activities. The calibration of the mortality model incorporates primary market quotes of annuities (unfortunately, there were no quotes of variable annuties available). Hence, we are able to analyze the differences between the survival-probabilities derived from mortality tables and the ones entering product pricing. In terms of the considered quotes the annuity providers seem to work with higher survival probabilities than the current mortality tables suggest. In this context, the premium resulting from the increased survival probabilities could be charged for taking the insureds' longevity risk. Another advantage of the presented approach is its flexibility. Since some empricial studies confirm the emergency fund hypothesis, i.e., the assumption that policyholders cancel their contracts due to personal financial distress, we demonstrate how the original scheme can be properly adjusted.

Due to a lack of data we are not able to calibrate the surrender model. Therefore, future research may focus on possible calibration approaches and model extensions. In this article the mortality risk premium is supposed to be constant with respect to the year a contract was issued and the age of the insured. The development of more sophisticated models covering the mortality risk premium together with an accordingly adjusted calibration method might be interesting as well. Finally, products like guaranteed minimum withdrawal benefits (GMWBs) and guaranteed minimum living benefits (GMLBs) as well as other types of guarantees like ratchets should be investigated.

Acknowledgments: Franz Ramsauer gratefully acknowledges the support from Pioneer Investments during his doctoral phase. Mikhail Krayzler acknowledges support from risklab during his doctoral studies. David Saunders and Marcos Escobar acknowledge support of the Natural Sciences and Engineering Research Council of Canada, in the form of Discovery Grants. 
Author Contributions: All authors contributed equally to this research work by providing new ideas, discussing existing results and sharing their profound knowledge in this field. In case of the derivation and implementation of the closed-form approximation as well as the model calibration F.R. did the most within the scope of [38].

Conflicts of Interest: The authors declare no conflict of interest. The founding sponsors had no role in the design of the study; in the collection, analyses, or interpretation of data; in the writing of the manuscript, and in the decision to publish the results.

\section{Abbreviations}

The following abbreviations are used in this manuscript:

$\begin{array}{ll}\text { ATM } & \begin{array}{l}\text { at-the-money } \\ \text { bn }\end{array} \\ \text { cDllion } \\ \text { CDF } & \text { cumulative distribution function } \\ \text { DAX } & \text { Deutscher Aktienindex } \\ \text { EURIBOR } & \text { Euro Interbank Offered Rate } \\ \text { GMAB } & \text { guaranteed minimum accumulation benefit } \\ \text { GMDB } & \text { guaranteed minimum death benefit } \\ \text { GMLB } & \text { guaranteed minimum living benefit } \\ \text { GMWB } & \text { guaranteed minimum withdrawal benefit } \\ \text { GMXB } & \text { guaranteed minimum benefit } \\ \text { MC } & \text { Monte Carlo Simulation } \\ \text { SB } & \text { surrender benefit } \\ \text { U.K. } & \text { United Kingdom } \\ \text { U.S. } & \text { United States } \\ \text { VA } & \text { Variable annuity }\end{array}$

\section{Appendix A. Required Distributions}

\section{Appendix A.1. Financial Market Model}

The closed-form approximation requires expectations and variances under the risk-neutral measure $\mathbb{Q}$, the forward measure $\mathbb{Q}^{T}$ in (12) and the equity measure $\mathbb{Q}^{S\left(t_{i}\right)}$ in (26). For all $0 \leq t \leq T$ we define:

$$
V(t)=\frac{\sigma_{r}^{2}}{a_{r}^{3}}\left[2 \exp \left(-a_{r} t\right)-\frac{1}{2} \exp \left(-2 a_{r} t\right)+a_{r} t-\frac{3}{2}\right] .
$$

Then, it follows from (1) that the stock return is normally distributed under $\mathbb{Q}$ with:

$$
\begin{aligned}
\mathbb{E}_{\mathbb{Q}}[Y(t)] & =\frac{1}{2} V(t)-\ln (P(0, t))-\frac{1}{2} \int_{0}^{t} \sigma_{S}^{2}(s) \mathrm{d} s \\
\operatorname{Var}_{\mathbb{Q}}[Y(t)] & =\int_{0}^{t} \sigma_{S}^{2}(s) \mathrm{d} s-\frac{2 \rho_{S r} \sigma_{r}}{a_{r}} \int_{0}^{t} \sigma_{S}(s)\left[\exp \left(a_{r}(s-t)\right)-1\right] \mathrm{d} s+V(t) .
\end{aligned}
$$

Using (12), the Girsanov theorem preserves the normal distribution and provides for all $0 \leq t \leq T$ :

$$
\begin{aligned}
\mathbb{E}_{\mathbb{Q}^{T}}[Y(t)] & =\rho_{S r} \frac{\sigma_{r}}{a_{r}} \int_{0}^{t} \sigma_{S}(s)\left[\exp \left(a_{r}(s-T)\right)-1\right] \mathrm{d} s-\frac{\sigma_{r}^{2}}{2 a_{r}^{3}}\left[a_{r} t+\frac{1}{2}\left(\exp \left(-2 a_{r} t\right)-1\right)\right]-\ln (P(0, t)) \\
& +\frac{\sigma_{r}^{2}}{2 a_{r}^{3}}\left[\exp \left(a_{r}(t-T)\right)+\exp \left(-a_{r}(t+T)\right)-2 \exp \left(-a_{r} T\right)\right]-\frac{1}{2} \int_{0}^{t} \sigma_{S}^{2}(s) \mathrm{d} s .
\end{aligned}
$$

As the performed measure change only adjusts the drift, the variances of $Y(t)$ under $\mathbb{Q}^{T}$ and $\mathbb{Q}$ coincide. For $0 \leq t \leq T$ the expectation $\mathbb{E}_{\mathbb{Q}^{S(t)}}[Y(t)]$ and the variance $\operatorname{Var}_{\mathbb{Q}^{S(t)}}[Y(t)]$ are stated in Theorem 3.1 in [6]. The rollup rate $\delta$ and the lower limit function $l(t), 0 \leq t \leq T$, are deterministic. Thus, for all $0 \leq t \leq T$ the preceding variances are the same for $\tilde{Y}(t)=Y(t)-\delta t$ and $\tilde{D}(t)=Y(t)-l(t)$. The expectations of $\tilde{Y}(t)$ and $\tilde{D}(t)$ are equal to the expectation of $Y(t)$ reduced by $\delta t$ and $l(t)$, respectively. 
For all $0 \leq t \leq T$ the decision criterion $D(t)$ is normally distributed with:

$$
\begin{aligned}
\mathbb{E}_{\mathbb{Q}}[D(t)] & =\frac{\sigma_{r}^{2}}{2 a_{r}^{3}}\left[a_{r} t+2\left(\exp \left(-a_{r} T\right)-\exp \left(a_{r}(t-T)\right)\right)\right]-\frac{1}{2} \int_{0}^{t} \sigma_{S}^{2}(s) \mathrm{d} s \\
& -\frac{\sigma_{r}^{2}}{4 a_{r}^{3}}\left[\exp \left(-2 a_{r} T\right)-\exp \left(2 a_{r}(t-T)\right)\right]-f(t)-\delta T-\ln (P(0, T)), \\
\operatorname{Var}_{\mathbb{Q}}[D(t)] & =\frac{\sigma_{r}^{2}}{2 a_{r}^{3}}\left[\exp \left(2 a_{r}(t-T)\right)-\exp \left(-2 a_{r} T\right)\right]+2 \rho_{S r} \frac{\sigma_{r}}{a_{r}} \int_{0}^{t} \sigma_{S}(s)\left(1-\exp \left(a_{r}(s-T)\right)\right) \mathrm{d} s \\
& +\frac{\sigma_{r}^{2}}{a_{r}^{3}}\left[a_{r} t-2\left(\exp \left(a_{r}(t-T)\right)-\exp \left(-a_{r} T\right)\right)\right]+\int_{0}^{t} \sigma_{S}^{2}(s) \mathrm{d} s .
\end{aligned}
$$

Again, a change of measure provides for the expectation under the forward measure $\mathbb{Q}^{T}$ :

$$
\begin{aligned}
\mathbb{E}_{\mathbb{Q}^{T}}[D(t)]= & \frac{\sigma_{r}^{2}}{4 a_{r}^{3}}\left[\exp \left(-2 a_{r} T\right)-\exp \left(2 a_{r}(t-T)\right)\right]-\frac{1}{2} \int_{0}^{t} \sigma_{S}^{2}(s) \mathrm{d} s-\delta T-\ln (P(0, T))-f(t) \\
& -\frac{\sigma_{r}^{2}}{2 a_{r}^{3}}\left[a_{r} t+2\left(\exp \left(-a_{r} T\right)-\exp \left(a_{r}(t-T)\right)\right)\right]-\rho_{S r} \frac{\sigma_{r}}{a_{r}} \int_{0}^{t} \sigma_{S}(s)\left(1-\exp \left(a_{r}(s-T)\right)\right) \mathrm{d} s .
\end{aligned}
$$

In the case of the variance no change occurs, that is, the variances under $\mathbb{Q}^{T}$ and $\mathbb{Q}$ coincide. Next, we have:

$$
\begin{aligned}
\operatorname{Cov}_{\mathbb{Q}^{T}}[D(t), Y(T)] & =\int_{0}^{t} \sigma_{S}^{2}(s) \mathrm{d} s+2 \rho_{S r} \frac{\sigma_{r}}{a_{r}} \int_{0}^{t} \sigma_{S}(s)\left(1-\exp \left(a_{r}(s-T)\right)\right) \mathrm{d} s \\
+ & \frac{\sigma_{r}^{2}}{a_{r}^{3}}\left[a_{r} t-\frac{1}{2}\left(\exp \left(-2 a_{r} T\right)-\exp \left(2 a_{r}(t-T)\right)\right)+2\left(\exp \left(-a_{r} T\right)-\exp \left(a_{r}(t-T)\right)\right)\right] .
\end{aligned}
$$

Finally, for $0 \leq t \leq T$ we obtain the following expectation of $D(t)$ under the equity measure $\mathbb{Q}^{S(T)}$ :

$$
\begin{gathered}
\mathbb{E}_{\mathbb{Q}^{S(T)}}[D(t)]=\frac{\sigma_{r}^{2}}{2 a_{r}^{3}}\left[a_{r} t+2\left(\exp \left(-a_{r} T\right)-\exp \left(a_{r}(t-T)\right)\right)\right]+\frac{1}{2} \int_{0}^{t} \sigma_{S}^{2}(s) \mathrm{d} s-\delta T-\ln (P(0, T)) \\
-\frac{\sigma_{r}^{2}}{4 a_{r}^{3}}\left[\exp \left(-2 a_{r} T\right)-\exp \left(2 a_{r}(t-T)\right)\right]+\rho_{S r} \frac{\sigma_{r}}{a_{r}} \int_{0}^{t} \sigma_{S}(s)\left(1-\exp \left(a_{r}(s-T)\right)\right) \mathrm{d} s-f(t) .
\end{gathered}
$$

As before, $\operatorname{Var}_{\mathbb{Q}^{S(T)}}[D(t)]$ remains unaffected by the measure change and thus it coincides with $\operatorname{Var}_{\mathbb{Q}}[D(t)]$

\section{Appendix A.2. Insurance Market Model}

Using Itô's Lemma as well as Equations (2) and (3), it can be shown that the mortality intensity at time $T$ is normally distributed with the moments $\mu_{\lambda}^{\mathbb{Q}}, \sigma_{\lambda}^{\mathbb{Q}}$ for the risk-neutral case and $\mu_{\lambda}^{\mathbb{P}}, \sigma_{\lambda}^{\mathbb{P}}$ for the real-world case:

$$
\begin{aligned}
\mu_{\lambda(T)}^{\mathbb{P}} & =\mathbb{E}_{\mathbb{P}}\left[\lambda_{T}(x+T)\right]=\lambda_{0}(x) \exp \left(-c_{3} T\right)+\frac{c_{1}}{c_{2}+c_{3}}\left(\exp \left(c_{2} T\right)-\exp \left(-c_{3} T\right)\right), \\
\left(\sigma_{\lambda(T)}^{\mathbb{P}}\right)^{2} & =\operatorname{Var}_{\mathbb{P}}\left[\lambda_{T}(x+T)\right]=\frac{c_{4}^{2}}{2 c_{5}+2 c_{3}}\left(\exp \left(2 c_{5} T\right)-\exp \left(-2 c_{3} T\right)\right), \\
\mu_{\lambda(T)}^{\mathbb{Q}} & =\mathbb{E}_{\mathbb{Q}}\left[\lambda_{T}(x+T)\right]=\lambda_{0}(x) \exp \left(-c_{3} T\right)+\frac{c_{1}}{c_{2}+c_{3}}\left(\exp \left(c_{2} T\right)-\exp \left(-c_{3} T\right)\right) \\
& -\frac{c_{4} \gamma_{m}}{c_{5}+c_{3}}\left(\exp \left(c_{5} T\right)-\exp \left(-c_{3} T\right)\right), \\
\left(\sigma_{\lambda(T)}^{\mathbb{Q}}\right)^{2} & =\operatorname{Var}_{\mathbb{Q}}\left[\lambda_{T}(x+T)\right]=\frac{c_{4}^{2}}{2 c_{5}+2 c_{3}}\left(\exp \left(2 c_{5} T\right)-\exp \left(-2 c_{3} T\right)\right) .
\end{aligned}
$$


Then, the probability of negative mortality intensity can be calculated analytically as:

$$
\mathbb{P}\left(\lambda_{T}(x+T)<0\right)=\mathbb{P}\left(\frac{\lambda_{T}(x+T)-\mu_{\lambda(T)}}{\sigma_{\lambda(T)}}<-\frac{\mu_{\lambda(T)}}{\sigma_{\lambda(T)}}\right)=\Phi\left(-\frac{\mu_{\lambda(T)}}{\sigma_{\lambda(T)}}\right)
$$

where $\Phi(\cdot)$ is the cumulative distribution function of the standard normal distribution and $\mu_{\lambda(T)}$ and $\sigma_{\lambda(T)}$ are the corresponding moments of the mortality intensity at time $T$ taken either under the risk-neutral or the real-world measure.

\section{Appendix B. Required Theorems}

Within this section we provide some general results which are important for the proofs in Appendix C.

Appendix B.1. Exponential of Truncated Univariate Gaussian

Assume $a \in \mathbb{R}$ and $b \in \mathbb{R} \backslash\{0\}$ and let $X$ be a normally distributed random variable on the probability space $(\Omega, \mathcal{F}, \mathbb{P})$ with mean $\mu$ and variance $\sigma^{2}$, i.e., $X \sim \mathcal{N}\left(\mu, \sigma^{2}\right)$. Then, we have that:

1. if $b>0$ :

$$
\begin{aligned}
\mathbb{E}_{\mathbb{P}}\left[\exp (a+b X) \mathbb{1}_{\{a+b X \geq 0\}}\right] & =\exp \left(a+b \mu+\frac{1}{2} b^{2} \sigma^{2}\right) \Phi\left(\frac{a / b+b \sigma^{2}+\mu}{\sigma}\right), \\
\mathbb{E}_{\mathbb{P}}\left[\exp (-a-b X) \mathbb{1}_{\{a+b X \geq 0\}}\right] & =\exp \left(-a-b \mu+\frac{1}{2} b^{2} \sigma^{2}\right) \Phi\left(\frac{a / b-b \sigma^{2}+\mu}{\sigma}\right),
\end{aligned}
$$

2. if $b<0$ :

$$
\begin{aligned}
\mathbb{E}_{\mathbb{P}}\left[\exp (a+b X) \mathbb{1}_{\{a+b X \geq 0\}}\right] & =\exp \left(a+b \mu+\frac{1}{2} b^{2} \sigma^{2}\right) \Phi\left(-\frac{a / b+b \sigma^{2}+\mu}{\sigma}\right) \\
\mathbb{E}_{\mathbb{P}}\left[\exp (-a-b X) \mathbb{1}_{\{a+b X \geq 0\}}\right] & =\exp \left(-a-b \mu+\frac{1}{2} b^{2} \sigma^{2}\right) \Phi\left(-\frac{a / b-b \sigma^{2}+\mu}{\sigma}\right) .
\end{aligned}
$$

Proof. For $b>0$ we obtain:

$$
\begin{aligned}
\mathbb{E}_{\mathbb{P}}\left[\exp (a+b X) \mathbb{1}_{\{a+b X \geq 0\}}\right]=\frac{1}{\sqrt{2 \pi} \sigma} \int_{-a / b}^{\infty} \exp (a+b x) \exp \left(-\frac{1}{2}\left(\frac{x-\mu}{\sigma}\right)^{2}\right) d x \\
=\exp \left(a+b \mu+\frac{1}{2} b^{2} \sigma^{2}\right) \frac{1}{\sqrt{2 \pi} \sigma} \int_{-a / b}^{\infty} \exp \left(-\frac{1}{2}\left(\frac{x-\left(b \sigma^{2}+\mu\right)}{\sigma}\right)^{2}\right) d x \\
=\exp \left(a+b \mu+\frac{1}{2} b^{2} \sigma^{2}\right)\left(1-\Phi\left(-\frac{a / b+b \sigma^{2}+\mu}{\sigma}\right)\right) \\
=\exp \left(a+b \mu+\frac{1}{2} b^{2} \sigma^{2}\right) \Phi\left(\frac{a / b+b \sigma^{2}+\mu}{\sigma}\right) .
\end{aligned}
$$

The remaining formulas can be similarly proved.

\section{Appendix B.2. Exponential of Truncated Multivariate Gaussian}

Let $(\Omega, \mathcal{F}, \mathbb{P})$ be a probability space and let $X:=\left(X_{1}, \ldots, X_{n}\right)^{\prime} \in \mathbb{R}^{n}$ be a Gaussian random variable with mean $\mu:=\left(\mu_{1}, \ldots, \mu_{n}\right)^{\prime}$ and covariance matrix $\Sigma$, i.e., $X \sim \mathcal{N}(\mu, \Sigma)$. For any lower limit 
$l:=\left(l_{1}, \ldots, l_{n}\right)^{\prime}$ and any transformation matrix $D \in \mathbb{R}^{n \times n}$ the moment generating function of the $D$-transformed random variable $X$ at $u:=\left(u_{1}, \ldots, u_{n}\right)^{\prime}$ called $M(u, \mu, \Sigma, l, D)$ is given by:

$$
M(u, \mu, \Sigma, l, D):=\mathbb{E}_{\mathbb{P}}\left[\exp \left(u^{\prime} D X\right) \mathbb{1}_{\{X \geq l\}}\right]=\mathbb{P}(\hat{X} \geq l-\mu) \exp \left(u^{\prime} D \mu+\frac{1}{2} u^{\prime} D \Sigma D^{\prime} u\right),
$$

where $\hat{X} \geq l-\mu$ holds component-by-component, i.e $\hat{X}_{i} \geq l_{i}-\mu_{i} \forall 1 \leq i \leq n$, and $\hat{X} \sim \mathcal{N}\left(\Sigma D^{\prime} u, \Sigma\right)$.

Proof. Similar to the steps in [39] we define $Z:=X-\mu$ and use that:

$$
-\frac{1}{2} z^{\prime} \Sigma^{-1} z+u^{\prime} D z=\frac{1}{2} u^{\prime} D \Sigma D^{\prime} u-\frac{1}{2}\left(z-\Sigma D^{\prime} u\right)^{\prime} \Sigma^{-1}\left(z-\Sigma D^{\prime} u\right)
$$

holds for all $z \in \mathbb{R}$. Then, we get:

$$
\begin{aligned}
M(u, \mu, \Sigma, l, D): & =\mathbb{E}_{\mathbb{P}}\left[\exp \left(u^{\prime} D X\right) \mathbb{1}_{\{X \geq l\}}\right] \\
& =\exp \left(u^{\prime} D \mu\right) \int_{l-\mu}^{\infty}\left(\frac{1}{2 \pi}\right)^{n / 2}\left(\frac{1}{|\Sigma|}\right)^{1 / 2} \exp \left(u^{\prime} D z-\frac{1}{2} z^{\prime} \Sigma^{-1} z\right) d z \\
& =\mathbb{P}(\hat{X} \geq l-\mu) \exp \left(u^{\prime} D \mu+\frac{1}{2} u^{\prime} D \Sigma D^{\prime} u\right)
\end{aligned}
$$

with $\hat{X} \sim \mathcal{N}\left(\Sigma D^{\prime} u, \Sigma\right)$.

\section{Appendix B.3. First Order Moments of Truncated Bivariate Gaussian}

Let $(\Omega, \mathcal{F}, \mathbb{P})$ be a probability space and let $(X, Y)^{\prime} \in \mathbb{R}^{2}$ be a Gaussian random variable with mean zero and standardized covariance matrix, i.e.,:

$$
\mu:=\left(\begin{array}{l}
0 \\
0
\end{array}\right) \quad \text { and } \quad \Sigma:=\left(\begin{array}{ll}
1 & \rho \\
\rho & 1
\end{array}\right) .
$$

Then, for any $h, k \in \mathbb{R}$ it follows:

$$
\begin{aligned}
& \mathbb{E}_{\mathbb{P}}\left[X \mathbb{1}_{\{X>h, Y>k\}}\right]=\varphi(h) \Phi\left(-\frac{k-\rho h}{\sqrt{1-\rho^{2}}}\right)+\rho \varphi(k) \Phi\left(-\frac{h-\rho k}{\sqrt{1-\rho^{2}}}\right), \\
& \mathbb{E}_{\mathbb{P}}\left[X \mathbb{1}_{\{X<h, Y<k\}}\right]=-\varphi(h) \Phi\left(\frac{k-\rho h}{\sqrt{1-\rho^{2}}}\right)-\rho \varphi(k) \Phi\left(\frac{h-\rho k}{\sqrt{1-\rho^{2}}}\right),
\end{aligned}
$$

with $\varphi(\cdot)$ denoting the probability density function of the univariate standard normal distribution.

Proof. The original proof of this claim is given in [40]. However, as there are some errors in that reference, we prove it here. For $h, k \in \mathbb{R}$ the linear transformation $x:=\sqrt{1-\rho^{2}} z+\rho y$ provides:

$$
\begin{aligned}
\mathbb{E}_{\mathbb{P}}\left[X \mathbb{1}_{\{X>h, Y>k\}}\right] & =\frac{1}{2 \pi \sqrt{1-\rho^{2}}} \int_{k}^{\infty} \int_{h}^{\infty} x \exp \left(-\frac{1}{2}\left(\frac{x^{2}-2 \rho x y+y^{2}}{1-\rho^{2}}\right)\right) \mathrm{d} x \mathrm{~d} y \\
& =\frac{1}{2 \pi} \int_{k}^{\infty} \int_{\frac{h-\rho y}{\sqrt{1-\rho^{2}}}}^{\infty}\left(z \sqrt{1-\rho^{2}}+\rho y\right) \exp \left(-\frac{1}{2}\left(z^{2}+y^{2}\right)\right) \mathrm{d} z \mathrm{~d} y \\
& =\frac{\sqrt{1-\rho^{2}}}{2 \pi} \int_{k}^{\infty}\left[-\exp \left(-\frac{1}{2} z^{2}\right)\right]_{\frac{h-\rho y}{\sqrt{1-\rho^{2}}}}^{\infty} \exp \left(-\frac{1}{2} y^{2}\right) \mathrm{d} y
\end{aligned}
$$




$$
\begin{aligned}
& +\frac{\rho}{2 \pi} \int_{\frac{h-\rho k}{\sqrt{1-\rho^{2}}}}^{\infty} \int_{k}^{\infty} y \exp \left(-\frac{1}{2} y^{2}\right) \mathrm{d} y \exp \left(-\frac{1}{2} z^{2}\right) \mathrm{d} z \\
& +\frac{\rho}{2 \pi} \int_{-\infty}^{\frac{h-\rho k}{\sqrt{1-\rho^{2}}}} \int_{\frac{h-\sqrt{1-\rho^{2}} z}{\rho}}^{\infty} y \exp \left(-\frac{1}{2} y^{2}\right) \mathrm{d} y \exp \left(-\frac{1}{2} z^{2}\right) \mathrm{d} z \\
& =\frac{\sqrt{1-\rho^{2}}}{2 \pi} \int_{k}^{\infty} \exp \left(-\frac{1}{2} \frac{y^{2}+h^{2}-2 h \rho y}{1-\rho^{2}}\right) d y+\rho \varphi(k) \Phi\left(-\frac{h-\rho k}{\sqrt{1-\rho^{2}}}\right) \\
& +\frac{\rho}{2 \pi} \int_{-\infty}^{\frac{h-\rho k}{\sqrt{1-\rho^{2}}}} \exp \left(-\frac{1}{2} \frac{h^{2}+z^{2}-2 h z \sqrt{1-\rho^{2}}}{\rho^{2}}\right) d z .
\end{aligned}
$$

After the linear transformation $y:=\frac{h-\sqrt{1-\rho^{2}} z}{\rho}$ has been applied to the last integral, the first and the last expression can be summarized, and we end up with:

$$
\mathbb{E}_{\mathbb{P}}\left[X \mathbb{1}_{\{X>h, Y>k\}}\right]=\frac{1}{2 \pi \sqrt{1-\rho^{2}}} \int_{k}^{\infty} \exp \left(-\frac{1}{2} \frac{y^{2}+h^{2}-2 h \rho y}{1-\rho^{2}}\right) d y+\rho \varphi(k) \Phi\left(-\frac{h-\rho k}{\sqrt{1-\rho^{2}}}\right) .
$$

The linear transformation $z:=\frac{y-h \rho}{\sqrt{1-\rho^{2}}}$ provides the assertion. The second result is similarly derived.

Let $(\tilde{X}, \tilde{Y})^{\prime} \in \mathbb{R}^{2}$ be a Gaussian random variable with mean $\tilde{\mu}$ and covariance matrix $\tilde{\Sigma}$ defined by:

$$
\tilde{\mu}:=\left(\begin{array}{l}
\mu_{X} \\
\mu_{Y}
\end{array}\right) \quad \text { and } \quad \tilde{\Sigma}:=\left(\begin{array}{cc}
\sigma_{X}^{2} & \rho \sigma_{X} \sigma_{Y} \\
\rho \sigma_{X} \sigma_{Y} & \sigma_{Y}^{2}
\end{array}\right)
$$

Then, the standardized Gaussian random variables are given by:

$$
X:=\frac{\tilde{X}-\mu_{X}}{\sigma_{X}} \quad \text { and } \quad Y:=\frac{\tilde{Y}-\mu_{Y}}{\sigma_{Y}}
$$

For any fixed $\tilde{h}, \tilde{k} \in \mathbb{R}$ we set:

$$
h:=\frac{\tilde{h}-\mu_{X}}{\sigma_{X}} \quad \text { and } \quad k:=\frac{\tilde{k}-\mu_{Y}}{\sigma_{Y}}
$$

and obtain:

$$
\begin{aligned}
& \mathbb{E}_{\mathbb{P}}\left[\tilde{X} \mathbb{1}_{\{\tilde{X}>\tilde{h}, \tilde{Y}>\tilde{k}\}}\right]=\sigma_{X} \mathbb{E}_{\mathbb{P}}\left[X \mathbb{1}_{\{X>h, Y>k\}}\right]+\mu_{X} \mathbb{P}(X>h, Y>k), \\
& \mathbb{E}_{\mathbb{P}}\left[\tilde{X} \mathbb{1}_{\{\tilde{X}<\tilde{h}, \tilde{Y}<\tilde{k}\}}\right]=\sigma_{X} \mathbb{E}_{\mathbb{P}}\left[X \mathbb{1}_{\{X<h, Y<k\}}\right]+\mu_{X} \mathbb{P}(X<h, Y<k),
\end{aligned}
$$

using the above expectations of the truncated, standardized Gaussian $(X, Y)^{\prime}$.

Proof. Follows directly from Appendix (B1) and the characteristics of the expectation.

Appendix B.4. Second Order Moments of Truncated Bivariate Gaussian

Let $(X, Y)^{\prime} \in \mathbb{R}^{2}$ be the standardized Gaussian of Appendix B.3 with $0<\rho<1$. For $h, k \in \mathbb{R}$ we define:

$$
l_{1}:=\frac{k-\rho h}{\sqrt{1-\rho^{2}}}, \quad l_{2}:=\frac{h-\rho k}{\sqrt{1-\rho^{2}}} \quad \text { and } \quad \bar{\Sigma}:=\left(\begin{array}{cc}
1 & -\sqrt{1-\rho^{2}} \\
-\sqrt{1-\rho^{2}} & 1
\end{array}\right)
$$


Then, we have that:

$$
\begin{aligned}
& \mathbb{E}_{\mathbb{P}}\left[X^{2} \mathbb{1}_{\{X>h, Y>k\}}\right]=\varphi(h)\left[h \Phi\left(-l_{1}\right)+\rho \sqrt{1-\rho^{2}} \varphi\left(l_{1}\right)\right]+\left(1-\rho^{2}\right) \mathbb{P}(X>h, Y>k) \\
& +\rho^{2} \Phi\left(-l_{2}\right)[\Phi(-k)+k \varphi(k)]+\rho^{2} \mathbb{P}\left(\bar{X}<-h, \bar{Y}<l_{2}\right), \\
& \mathbb{E}_{\mathbb{P}}\left[X Y \mathbb{1}_{\{X>h, Y>k\}}\right]=\varphi(h)\left[\sqrt{1-\rho^{2}} \varphi\left(l_{1}\right)+h \rho \Phi\left(-l_{1}\right)\right]+\rho \Phi\left(-l_{2}\right)[k \varphi(k)+\Phi(-k)] \\
& +\rho \mathbb{P}\left(\bar{X}<-h, \bar{Y}<l_{2}\right), \\
& \mathbb{E}_{\mathbb{P}}\left[X^{2} \mathbb{1}_{\{X<h, Y<k\}}\right]=\varphi(h)\left[\rho \sqrt{1-\rho^{2}} \varphi\left(l_{1}\right)-h \Phi\left(l_{1}\right)\right]+\left(1-\rho^{2}\right) \mathbb{P}(X<h, Y<k) \\
& +\rho^{2} \Phi\left(l_{2}\right)[\Phi(k)-k \varphi(k)]+\rho^{2} \mathbb{P}\left(\bar{X}<h, \bar{Y}<-l_{2}\right), \\
& \mathbb{E}_{\mathbb{P}}\left[X Y \mathbb{1}_{\{X<h, Y<k\}}\right]=\varphi(h)\left[\sqrt{1-\rho^{2}} \varphi\left(l_{1}\right)-h \rho \Phi\left(l_{1}\right)\right]+\rho \Phi\left(l_{2}\right)[\Phi(k)-k \varphi(k)] \\
& +\rho \mathbb{P}\left(\bar{X}<h, \bar{Y}<-l_{2}\right),
\end{aligned}
$$

where $(\bar{X}, \bar{Y})^{\prime}$ denotes a bivariate Gaussian random variable with mean zero and variance $\bar{\Sigma}$.

Proof. The work in [40] shows for $h, k \in \mathbb{R}$ :

$$
\begin{aligned}
\mathbb{E}_{\mathbb{P}}\left[X^{2} \mathbb{1}_{\{X>h, Y>k\}}\right] & =\int_{k}^{\infty} \exp \left(-\frac{1}{2}\left(\frac{h^{2}-2 \rho h y+y^{2}}{1-\rho^{2}}\right)\right) \frac{\sqrt{1-\rho^{2}}(h+\rho y)}{2 \pi} d y \\
& +\int_{k}^{\infty} \exp \left(-\frac{1}{2} y^{2}\right)\left(\frac{1-\rho^{2}+\rho^{2} y^{2}}{2 \pi}\right) \int_{\frac{h-\rho y}{\sqrt{1-\rho^{2}}}}^{\infty} \exp \left(-\frac{1}{2}\right) d z d y .
\end{aligned}
$$

Next, we apply $y:=\sqrt{1-\rho^{2}} z+h \rho$ to the first integral, $x:=\sqrt{1-\rho^{2}} z+\rho y$ to the first two summands of the second integral and change the order of integration for the third term of the second integral:

$$
\begin{aligned}
\mathbb{E}_{\mathbb{P}}\left[X^{2} \mathbb{1}_{\{X>h, Y>k\}}\right] & =\int_{\frac{k-h \rho}{\sqrt{1-\rho^{2}}}}^{\infty} \frac{\left(1-\rho^{2}\right)\left(h+\rho \sqrt{1-\rho^{2}} z+\rho^{2} h\right)}{2 \pi} \exp \left(-\frac{1}{2}\left(z^{2}+h^{2}\right)\right) d z \\
& +\frac{\sqrt{1-\rho^{2}}}{2 \pi} \int_{k}^{\infty} \int_{h}^{\infty} \exp \left(-\frac{1}{2} \frac{x^{2}-2 \rho x y+y^{2}}{1-\rho^{2}}\right) d x d y \\
& +\frac{\rho^{2}}{2 \pi} \int_{\frac{h-\rho k}{\sqrt{1-\rho^{2}}}}^{\infty} \int_{k}^{\infty} y^{2} \mathrm{e}^{-\frac{1}{2} y^{2}} d y \exp \left(-\frac{1}{2} z^{2}\right) d z \\
& +\frac{\rho^{2}}{2 \pi} \int_{-\infty}^{\frac{h-\rho k}{\sqrt{1-\rho^{2}}}} \int_{\frac{h-\sqrt{1-\rho^{2} z}}{\rho}}^{\infty} y^{2} \exp \left(-\frac{1}{2} y^{2}\right) d y \exp \left(-\frac{1}{2} z^{2}\right) d z .
\end{aligned}
$$

Using (partial) integration we get after the substitution of $x:=\sqrt{1-\rho^{2}} z+\rho y$ :

$$
\begin{aligned}
\mathbb{E}_{\mathbb{P}}\left[X^{2} \mathbb{1}_{\{X>h, Y>k\}}\right] & =\varphi(h)\left[\left(1-\rho^{2}\right) h\left(1+\rho^{2}\right) \Phi\left(-l_{1}\right)+\rho\left(1-\rho^{2}\right)^{3 / 2} \varphi\left(l_{1}\right)\right] \\
& +\left(1-\rho^{2}\right) \mathbb{P}(X>h, Y>k)+\rho^{2} \Phi\left(-l_{2}\right)[k \varphi(k)+\Phi(-k)] \\
& +\frac{\rho}{2 \pi} \int_{-\infty}^{l_{2}}\left(h-\sqrt{1-\rho^{2} z}\right) \exp \left(-\frac{1}{2} \frac{h^{2}-2 h z \sqrt{1-\rho^{2}}+z^{2}}{\rho^{2}}\right) \mathrm{d} z \\
& +\frac{\rho}{2 \pi} \int_{-\infty}^{l_{2}} \int_{h}^{\infty} \exp \left(-\frac{1}{2} \frac{x^{2}-2 x z \sqrt{1-\rho^{2}}+z^{2}}{\rho^{2}}\right) d x d z .
\end{aligned}
$$


If $w:=\frac{1}{\rho}\left(z-\sqrt{1-\rho^{2}} h\right)$ is used for the first integral and the direction of integrating $x$ (second integral) is turned by $\tilde{x}:=-x$, summarizing all terms yields the statement. Similarly, the remaining can be proved.

Let $(\tilde{X}, \tilde{Y})^{\prime} \in \mathbb{R}^{2}$ be the Gaussian of Appendix B.3 with $0<\rho<1$ and let $(X, Y)^{\prime}$ be its standardized counterpart in Appendix (B1). For any $\tilde{h}, \tilde{k} \in \mathbb{R}$ let $h$ and $k$ be given by Appendix (B2). Then, it holds that:

$$
\begin{aligned}
\mathbb{E}_{\mathbb{P}}\left[\tilde{X}^{2} \mathbb{1}_{\{\tilde{X}>\tilde{h}, \tilde{Y}>\tilde{k}\}}\right] & =\sigma_{X}^{2} \mathbb{E}_{\mathbb{P}}\left[X^{2} \mathbb{1}_{\{X>h, Y>k\}}\right]+2 \sigma_{X} \mu_{X} \mathbb{E}_{\mathbb{P}}\left[X \mathbb{1}_{\{X>h, Y>k\}}\right]+\mu_{X}^{2} \mathbb{P}(X>h, Y>k), \\
\mathbb{E}_{\mathbb{P}}\left[\tilde{X} \tilde{Y} \mathbb{1}_{\{\tilde{X}>\tilde{h}, \tilde{Y}>\tilde{k}\}}\right] & =\sigma_{X} \sigma_{Y} \mathbb{E}_{\mathbb{P}}\left[X Y \mathbb{1}_{\{X>h, Y>k\}}\right]+\sigma_{X} \mu_{Y} \mathbb{E}_{\mathbb{P}}\left[X \mathbb{1}_{\{X>h, Y>k\}}\right] \\
& +\sigma_{Y} \mu_{X} \mathbb{E}_{\mathbb{P}}\left[Y \mathbb{1}_{\{X>h, Y>k\}}\right]+\mu_{X} \mu_{Y} \mathbb{P}(X>h, Y>k) .
\end{aligned}
$$

In case of upper truncation $\mathbb{1}_{\{X>h, Y>k\}}$ has to be replaced by $\mathbb{1}_{\{X<h, Y<k\}}$.

Proof. Follows directly from Appendix (B1) and the characteristics of the expectation.

\section{Appendix C. Approximation Proofs}

Appendix C.1. Proof of $S_{11}^{\mathbb{Q}^{T}}(D, \alpha, \beta, \mathbf{t})$

The $S_{11}^{\mathbb{Q}^{T}}(D, \alpha, \beta, \mathbf{t})$ in (15) is equal to:

$$
\begin{aligned}
S_{11}^{\mathbb{Q}^{T}}(D, \alpha, \beta, \mathbf{t}) & =1+\sum_{i=1}^{K} \mathbb{E}_{\mathbb{Q}^{T}}\left[\exp \left(-\beta \Delta t_{i} D\left(t_{i}\right)\right) \mathbb{1}_{\left\{0<D\left(t_{i}\right)\right\}}\right]-\sum_{i=1}^{K} \mathbb{E}_{\mathbb{Q}^{T}}\left[\exp \left(-\beta \Delta t_{i} D\left(t_{i}\right)\right) \mathbb{1}_{\left\{D\left(t_{i}\right) \geq \alpha\right\}}\right] \\
& -\sum_{i=1}^{K} \mathbb{Q}^{T}\left(0<D\left(t_{i}\right)<\alpha\right)+\sum_{i=1}^{K}\left(\exp \left(-\beta \alpha \Delta t_{i}\right)-1\right) \mathbb{Q}^{T}\left(D\left(t_{i}\right) \geq \alpha\right) .
\end{aligned}
$$

Since $\alpha$ and $\beta$ are non-negative, we have that:

$$
\begin{aligned}
S_{11}^{\mathbb{Q}^{T}}(D, \alpha, \beta, \mathbf{t}) & =1+\sum_{i=1}^{K} \mathbb{E}_{\mathbb{Q}^{T}}\left[\exp \left(-\beta \Delta t_{i} D\left(t_{i}\right)\right) \mathbb{1}_{\left\{0<\beta \Delta t_{i} D\left(t_{i}\right)\right\}}\right] \\
& -\sum_{i=1}^{K} \exp \left(-\alpha \beta \Delta t_{i}\right) \mathbb{E}_{\mathbb{Q}^{T}}\left[\exp \left(-\beta \Delta t_{i}\left(D\left(t_{i}\right)-\alpha\right)\right) \mathbb{1}_{\left\{D\left(t_{i}\right)-\alpha \geq 0\right\}}\right] \\
& -\sum_{i=1}^{K}\left(\mathbb{Q}^{T}\left(D\left(t_{i}\right)<\alpha\right)-\mathbb{Q}^{T}\left(D\left(t_{i}\right) \leq 0\right)\right) \\
& +\sum_{i=1}^{K}\left(\exp \left(-\beta \alpha \Delta t_{i}\right)-1\right)\left(1-\mathbb{Q}^{T}\left(D\left(t_{i}\right)<\alpha\right)\right)
\end{aligned}
$$

with the help of Appendix B.1 we obtain that:

$$
\begin{aligned}
S_{11}^{\mathbb{Q}^{T}}(D, \alpha, \beta, \mathbf{t})= & 1+\sum_{i=1}^{K}\left[\exp \left(-\beta \Delta t_{i} \mathbb{E}_{\mathbb{Q}^{T}}\left[D\left(t_{i}\right)\right]+\frac{1}{2} \beta^{2} \Delta t_{i}^{2} \operatorname{Var}_{\mathbb{Q}^{T}}\left[D\left(t_{i}\right)\right]\right)\right. \\
& \left.\cdot \Phi\left(\frac{\mathbb{E}_{\mathbb{Q}^{T}}\left[D\left(t_{i}\right)\right]-\beta \Delta t_{i} \operatorname{Var}_{\mathbb{Q}^{T}}\left[D\left(t_{i}\right)\right]}{\sqrt{\operatorname{Var}_{\mathbb{Q}^{T}}\left[D\left(t_{i}\right)\right]}}\right)\right] \\
- & \sum_{i=1}^{K}\left[\exp \left(-\alpha \beta \Delta t_{i}-\beta \Delta t_{i} \mathbb{E}_{\mathbb{Q}^{T}}\left[D\left(t_{i}\right)-\alpha\right]+\frac{1}{2} \beta^{2} \Delta t_{i}^{2} \operatorname{Var}_{\mathbb{Q}^{T}}\left[D\left(t_{i}\right)-\alpha\right]\right)\right.
\end{aligned}
$$




$$
\begin{gathered}
\left.\cdot \Phi\left(\frac{\mathbb{E}_{\mathbb{Q}^{T}}\left[D\left(t_{i}\right)-\alpha\right]-\beta \Delta t_{i} \operatorname{Var}_{\mathbb{Q}^{T}}\left[D\left(t_{i}\right)-\alpha\right]}{\sqrt{\operatorname{Var}_{\mathbb{Q}^{T}}\left[D\left(t_{i}\right)-\alpha\right]}}\right)\right] \\
-\sum_{i=1}^{K}\left[\Phi\left(\frac{\alpha-\mathbb{E}_{\mathbb{Q}^{T}}\left[D\left(t_{i}\right)\right]}{\sqrt{\operatorname{Var}_{\mathbb{Q}^{T}}\left[D\left(t_{i}\right)\right]}}\right)-\Phi\left(\frac{-\mathbb{E}_{\mathbb{Q}^{T}}\left[D\left(t_{i}\right)\right]}{\sqrt{\operatorname{Var}_{\mathbb{Q}^{T}}\left[D\left(t_{i}\right)\right]}}\right)\right] \\
+\sum_{i=1}^{K}\left(\exp \left(-\alpha \beta \Delta t_{i}\right)-1\right)\left(1-\Phi\left(\frac{\alpha-\mathbb{E}_{\mathbb{Q}^{T}}\left[D\left(t_{i}\right)\right]}{\sqrt{\operatorname{Var}_{\mathbb{Q}^{T}}\left[D\left(t_{i}\right)\right]}}\right)\right) .
\end{gathered}
$$

Summarizing terms results in:

$$
\begin{aligned}
& S_{11}^{\mathbb{Q}^{T}}(D, \alpha, \beta, \mathbf{t})=1+\sum_{i=1}^{K}\left[\exp \left(-\beta \Delta t_{i} \mathbb{E}_{\mathbb{Q}^{T}}\left[D\left(t_{i}\right)\right]+\frac{1}{2} \beta^{2} \Delta t_{i}^{2} \operatorname{Var}_{\mathbb{Q}^{T}}\left[D\left(t_{i}\right)\right]\right)\right. \\
& \cdot\left(\Phi\left(\frac{\mathbb{E}_{\mathbb{Q}^{T}}\left[D\left(t_{i}\right)\right]-\beta \Delta t_{i} \operatorname{Var}_{\mathbb{Q}^{T}}\left[D\left(t_{i}\right)\right]}{\sqrt{\operatorname{Var}_{\mathbb{Q}^{T}}\left[D\left(t_{i}\right)\right]}}\right)\right. \\
& \left.\left.-\Phi\left(\frac{\mathbb{E}_{\mathbb{Q}^{T}}\left[D\left(t_{i}\right)\right]-\alpha-\beta \Delta t_{i} \operatorname{Var}_{\mathbb{Q}^{T}}\left[D\left(t_{i}\right)\right]}{\sqrt{\operatorname{Var}_{\mathbb{Q}^{T}}\left[D\left(t_{i}\right)\right]}}\right)\right)\right] \\
& -\sum_{i=1}^{K}\left[\Phi\left(\frac{\alpha-\mathbb{E}_{\mathbb{Q}^{T}}\left[D\left(t_{i}\right)\right]}{\sqrt{\operatorname{Var}_{\mathbb{Q}^{T}}\left[D\left(t_{i}\right)\right]}}\right)-\Phi\left(\frac{-\mathbb{E}_{\mathbb{Q}^{T}}\left[D\left(t_{i}\right)\right]}{\sqrt{\operatorname{Var}_{\mathbb{Q}^{T}}\left[D\left(t_{i}\right)\right]}}\right)\right] \\
& +\sum_{i=1}^{K}\left(\exp \left(-\alpha \beta \Delta t_{i}\right)-1\right)\left(1-\Phi\left(\frac{\alpha-\mathbb{E}_{\mathbb{Q}^{T}}\left[D\left(t_{i}\right)\right]}{\sqrt{\operatorname{Var}_{\mathbb{Q}^{T}}\left[D\left(t_{i}\right)\right]}}\right)\right)
\end{aligned}
$$

Finally, we use the definitions of $h_{1}\left(\beta_{l}, D, t_{i}\right)$ and $h_{2}\left(\alpha_{l}, \beta_{l}, D, t_{i}\right)$ in (19) to derive (20).

Appendix C.2. Proof of $S_{2}^{\mathbb{Q}^{T}}(D, \alpha, \beta, \mathbf{t})$

At first, we get rid of the products in $S_{2}^{\mathbb{Q}^{T}}(D, \tilde{Y}, \alpha, \beta, \mathbf{t})$ in (18) and properly split the indicator sets. For the first expression we use Appendix B.1. Then, we summarize the $\mathbb{Q}^{T}$-probabilities and so, we get:

$$
\begin{aligned}
S_{2}^{\mathbb{Q}^{T}}(D, \tilde{Y}, \alpha, \beta, \mathbf{t}) & =\exp \left(\mathbb{E}_{\mathbb{Q}^{T}}[\tilde{Y}(T)]+\frac{1}{2} \mathbb{V a r}_{\mathbb{Q}^{T}}[\tilde{Y}(T)]\right) \Phi\left(\frac{\mathbb{E}_{\mathbb{Q}^{T}}[\tilde{Y}(T)]+\operatorname{Var}_{\mathbb{Q}^{T}}[\tilde{Y}(T)]}{\sqrt{\operatorname{Var}_{\mathbb{Q}^{T}}[\tilde{Y}(T)]}}\right) \\
& +\sum_{i=1}^{K} \mathbb{E}_{\mathbb{Q}^{T}}\left[\exp \left(-\beta \Delta t_{i} D\left(t_{i}\right)+\tilde{Y}(T)\right) \mathbb{1}_{\left\{0<D\left(t_{i}\right)\right\}} \mathbb{1}_{\{\tilde{Y}(T) \geq 0\}}\right] \\
& -\sum_{i=1}^{K} \mathbb{E}_{\mathbb{Q}^{T}}\left[\exp \left(-\beta \Delta t_{i} D\left(t_{i}\right)+\tilde{Y}(T)\right) \mathbb{1}_{\left\{\alpha \leq D\left(t_{i}\right)\right\}} \mathbb{1}_{\{\tilde{Y}(T) \geq 0\}}\right] \\
& -\sum_{i=1}^{K} \mathbb{E}_{\mathbb{Q}^{T}}\left[\exp \left(-\beta \Delta t_{i} D\left(t_{i}\right)\right) \mathbb{1}_{\left\{0<D\left(t_{i}\right)\right\}} \mathbb{1}_{\{\tilde{Y}(T) \geq 0\}}\right] \\
& +\sum_{i=1}^{K} \mathbb{E}_{\mathbb{Q}^{T}}\left[\exp \left(-\beta \Delta t_{i} D\left(t_{i}\right)\right) \mathbb{1}_{\left\{\alpha \leq D\left(t_{i}\right)\right\}} \mathbb{1}_{\{\tilde{Y}(T) \geq 0\}}\right] \\
& -\sum_{i=1}^{K} \mathbb{E}_{\mathbb{Q}^{T}}\left[\exp (\tilde{Y}(T)) \mathbb{1}_{\left\{0<D\left(t_{i}\right)\right\}} \mathbb{1}_{\{\tilde{Y}(T) \geq 0\}}\right]
\end{aligned}
$$




$$
\begin{aligned}
& +\sum_{i=1}^{K} \mathbb{E}_{\mathbb{Q}^{T}}\left[\exp (\tilde{Y}(T)) \mathbb{1}_{\left\{\alpha \leq D\left(t_{i}\right)\right\}} \mathbb{1}_{\{\tilde{Y}(T) \geq 0\}}\right] \\
& +\sum_{i=1}^{K} \mathbb{Q}^{T}\left(0<D\left(t_{i}\right), \tilde{Y}(T) \geq 0\right) \\
& +\sum_{i=1}^{K} \exp \left(-\beta \alpha \Delta t_{i}\right) \mathbb{E}_{\mathbb{Q}^{T}}\left[\exp (\tilde{Y}(T)) \mathbb{1}_{\left\{D\left(t_{i}\right) \geq \alpha\right\}} \mathbb{1}_{\{\tilde{Y}(T) \geq 0\}}\right] \\
& -\sum_{i=1}^{K} \exp \left(-\beta \alpha \Delta t_{i}\right) \mathbb{Q}^{T}\left(D\left(t_{i}\right) \geq \alpha, \tilde{Y}(T) \geq 0\right) \\
& -\sum_{i=1}^{K} \mathbb{E}_{\mathbb{Q}^{T}}\left[\exp (\tilde{Y}(T)) \mathbb{1}_{\left\{D\left(t_{i}\right) \geq \alpha\right\}} \mathbb{1}_{\{Y(T) \geq 0\}}\right]-\left(1-\mathbb{Q}^{T}(\tilde{Y}(T)<0)\right)
\end{aligned}
$$

Using the notation in (23) and Appendix B.2 we obtain (24).

\section{References}

1. Bacinello, A.; Millossovich, P.; Olivieri, A.; Pitacco, E. Variable annuities: A unifying valuation approach. Insur. Math. Econ. 2011, 49, 285-297.

2. Ledlie, M.; Corry, D.; Finkelstein, G.; Ritchie, A.; Su, K.; Wilson, D. Variable Annuities. Br. Actuar. J. 2008, 14, 327-389.

3. Gatzert, N. The secondary market for life insurance in the United Kingdom, Germany, and the United States: Comparison and overview. Risk Manag. Insur. Rev. 2010, 13, 279-301.

4. Shevchenko, P.; Luo, X. A unified pricing of variable annuity guarantees under the optimal stochastic control framework. Risks 2016, 4, 22, doi:10.3390/risks4030022.

5. Knoller, C.; Kraut, G.; Schoenmaekers, P. On the propensity to surrender a variable annuity contract. J. Risk Insur. 2015, doi:10.1111/jori.12076.

6. Krayzler, M.; Zagst, R.; Brunner, B. Closed-form solutions for guaranteed minimum accumulation and death benefits. Eur. Actuar. J. 2016, 6, 197-231.

7. Tsai, C.; Kuo, W.; Chen, W. Early surrender and the distribution of policy reserves. Insur. Math. Econ. 2002, 31, 429-445.

8. Kuo, W.; Tsai, C.; Chen, W. An empirical study on the lapse rate: The cointegration approach. J. Risk Insur. 2003, 70, 489-508.

9. Outreville, A. Whole-life insurance lapse rates and the emergency fund hypothesis. Insur. Math. Econ. 1990, 9, 249-255.

10. Kim, C. Modeling surrender and lapse rates with economic variables. N. Am. Actuar. J. 2005, 9, 56-70.

11. Jiang, S. Voluntary termination of life insurance policies: Evidence from the U.S. market. N. Am. Actuar. J. 2010, 14, 369-380.

12. Kiesenbauer, D. Main determinants of lapse in the German life insurance industry. N. Am. Actuar. J. 2012, $16,52-73$.

13. Kent, J.; Ed, M. Dynamic Policyholder Behaviour; Presentation; Staple Inn Actuarial Society: London, UK, 2008.

14. Eling, M.; Kiesenbauer, D. Does surplus participation reflect market discipline? An analysis of the German life insurance market. J. Financ. Serv. Res. 2012, 42, 1-27.

15. Bacinello, A. Fair valuation of a guaranteed life insurance participating contract embedding a surrender option. J. Risk Insur. 2003, 70, 461-487.

16. Bacinello, A. Endogenous model of surrender conditions in equity-linked life insurance. Insur. Math. Econ. 2005, 37, 270-296.

17. Milevsky, M.; Salisbury, T. Financial valuation of guaranteed minimum withdrawal benefits. Insur. Math. Econ. 2006, 38, 21-38.

18. Chen, Z.; Vetzal, K.; Forsyth, P. The effect of modeling parameters on the value of GMWB guarantees. Insur. Math. Econ. 2008, 43, 165-173. 
19. Kling, A.; Ruez, F.; Ruß, J. The impact of stochastic volatility on pricing, hedging, and hedge efficiency of variable annuity guarantees. ASTIN Bull. 2011, 41, 511-545.

20. Moore, K. Optimal surrender strategies for equity-indexed annuity investors. Insur. Math. Econ. 2009, 44, 1-18.

21. Moenig, T.; Bauer, D. Revisiting the risk-neutral approach to optimal policyholder behavior: A study of withdrawal guarantees in variable annuities. Rev. Financ. 2015, doi:10.1093/rof/rfv018.

22. Albizzati, M.; Geman, H. Interest rate risk management and valuation of the surrender option in life insurance policies. J. Risk Insur. 1994, 61, 616-637.

23. Mudavanhu, B.; Zhuo, J. Valuing Guaranteed Minimum Death Benefits in Variable Annuities and the Option to Lapse. Working Paper, 2002.

24. Kolkiewicz, A.; Tan, K. Unit-linked life insurance contracts with lapse rates dependent on economic factors. Ann. Actuar. Sci. 2006, 1, 49-78.

25. De Giovanni, D. Lapse rate modeling: A rational expectation approach. Scand. Actuar. J. 2010, $2010,56-67$.

26. Loisel, S.; Milhaud, X. From deterministic to stochastic surrender risk models: Impact of correlation crises on economic capital. Eur. J. Oper. Res. 2011, 214, 348-357.

27. Hull, J.; White, A. Numerical procedures for implementing term structure models I: Single-factor models. J. Deriv. 1994, 2, 7-16.

28. Dahl, M. Stochastic mortality in life insurance: Market reserves and mortality-linked insurance contracts. Insur. Math. Econ. 2004, 35, 113-136.

29. Dahl, M.; Møller, T. Valuation and hedging of life insurance liabilities with systematic mortality risk. Insur. Math. Econ. 2006, 39, 193-217.

30. Biffis, E. Affine processes for dynamic mortality and actuarial valuations. Insur. Math. Econ. 2005, 37, 443-468.

31. Harrison, D. Is failure imminent for the United Kingdom's annuity market? Pensions 2012, 17, 71-79.

32. Biffis, E.; Denuit, M.; Devolder, P. Stochastic mortality under measure changes. Scand. Actuar. J. 2010, 2010, 284-311.

33. Lando, D. On cox processes and credit risky securities. Rev. Deriv. Res. 1998, 2, 99-120.

34. Duffie, D.; Pan, J.; Singleton, K. Transform analysis and asset pricing for affine jump-diffusions. Econometrica 2000, 68, 1343-1376.

35. Flanders, H. Differentiation under the integral sign. Am. Math. Mon. 1973, 80, 615-627.

36. Zagst, R. Interest Rate Management; Springer Finance: Berlin, Germany, 2002.

37. Cannon, E.; Tonks, I. UK annuity price series, 1957-2002. Financ. Hist. Rev. 2004, 11, 165-196.

38. Ramsauer, F. Pricing of Variable Annuities-Incorporation of Policyholder Behavior. Master's Thesis, Technical University of Munich, München, Germany, 2013.

39. Horrace, W. Some results on the multivariate truncated normal distribution. J. Multivar. Anal. 2005, 94, 209-221.

40. Rosenbaum, S. Moments of a truncated bivariate normal distribution. J. R. Stat. Soc. Ser. B 1961, 23, 405-408.

(C) 2016 by the authors; licensee MDPI, Basel, Switzerland. This article is an open access article distributed under the terms and conditions of the Creative Commons Attribution (CC-BY) license (http:/ / creativecommons.org/licenses/by/4.0/). 